\title{
Multispikes and Synchronization in a Large Neural Network with Temporal Delays
}

\section{Jan Karbowski}

Center for Biodynamics, Department of Mathematics, Boston University, Boston, MA 02215, U.S.A., and Center for Theoretical Physics, Polish Academy of Sciences, 02-668 Warsaw, Poland

\section{Nancy Kopell}

Center for Biodynamics, Department of Mathematics, Boston University, Boston, MA 02115, U.S.A.

Coherent rhythms in the gamma frequency range are ubiquitous in the nervous system and thought to be important in a variety of cognitive activities. Such rhythms are known to be able to synchronize with millisecond precision across distances with significant conduction delay; it is mysterious how this can operate in a setting in which cells receive many inputs over a range of time. Here we analyze a version of mechanism, previously proposed, that the synchronization in the CA1 region of the hippocampus depends on the firing of "doublets" by the interneurons. Using a network of local circuits that are arranged in a possibly disordered lattice, we determine the conditions on parameters for existence and stability of synchronous solutions in which the inhibitory interneurons fire single spikes, doublets, or triplets per cycle. We show that the synchronous solution is only marginally stable if the interneurons fire singlets. If they fire doublets, the synchronous state is asymptotically stable in a larger subset of parameter space than if they fire triplets. An unexpected finding is that a small amount of disorder in the lattice structure enlarges the parameter regime in which the doublet solution is stable. Synaptic noise reduces the regime in which the doublet configuration is stable, but only weakly.

\section{Introduction}

Coherent rhythms in the gamma range of frequencies $(30-80 \mathrm{~Hz})$ have been found in many parts of the cortex (Gray, Konig, Engel, \& Singel, 1989; Llinas \& Ribary, 1993; Bragin et al., 1995; Singer \& Gray, 1995; Steriade, Amzica, \& Contreras, 1996) and are hypothesized to be important in the creation of cell assemblies (Engel, Konig, \& Singer, 1991; Singer \& Gray, 1995; Vaadia et al., 1995; Stopfer, Bhagavan, Smith, \& Laurent, 1997) and in cognitive function (Bressler, Coppola, \& Nakamura, 1993; Joliot, Ribary, \& Llinas, 1994; Fries, Roelfsma, Engel, Konig, \& Singer, 1997). Coherent rhythms with no 
phase lags among the participating cells have a potentially important role to play in plasticity, since synchronous activity is known to encourage the strengthening of mutual connections (Hebb, 1949; Ahissar et al., 1992; Bliss \& Collingride, 1993; Magee \& Johnston, 1997; Markram, Lubke, Frotscher, \& Sakmann, 1997); though synchronous behavior is possible without rhythms, for the visual cortex it has been reported that such cells having a distance of more than $2 \mathrm{~mm}$ synchronize their activity only in the presence of rhythms (Konig, Engel, \& Singer, 1995). In addition, coherent activity of a set of cells potentiates their effects downstream in the processing.

Synchronization is known to exist between areas of the neocortex that are widely separated (Engel, Kreiter, Konig, \& Singer, 1991; Bressler et al., 1993; Konig et al., 1995; Roelfsema, Engel, Konig, \& Singer, 1997). In hippocampal slice preparations, synchronization of local field potentials and single unit recordings in the gamma frequency range are observed with areas up to several millimeters apart synchronized within a millesecond in spite of conduction delays that are many times that long (Andersen, Silfvenius, Sundberg, Sveen, \& Wigstrom, 1978; Traub, Whittington, Stanford, \& Jefferys, 1996). This article takes up the question of how such synchronization over long distances can take place in a stable manner.

For networks of spiking neurons, this question has been addressed in two closely related articles. In a pioneering article about the CA1 region of the hippocampus, Traub, Whittington, Stanford, and Jefferys (1996) used large-scale simulations of models containing excitatory pyramidal cells and inhibitory interneurons. They showed that synchronization was invariably accompanied by double spikes (or "doublets") per cycle in many of the interneurons. The role of the doublets in the synchronization at a pair of separated sites was deduced by Ermentrout and Kopell (1998). In a much reduced setting, the latter showed that in order for the doublets to aid in the synchronization process, it was important that the first spike of the doublet be due to excitation from the local pyramidal cells and the second spike be due to excitation from the remote site. The delay between the two spikes of a doublet encoded not just the conduction and synaptic delays but also the longer time to spiking that a cell experiences when it is not fully recovered from a previous spike. Ermentrout and Kopell (1998) demonstrated that the timing between the spikes could be used by the system to adjust the timing of the pyramidal cells automatically on the next cycle, in such a way as to make the coherent rhythm stable. (Related articles not as directly relevant are referred to in section 5.) The hypothesis that the first spike be due to excitation leads to a phase lag between the local excitatory and inhibitory cells. The value of this lag plays no role in the analysis here or in Ermentrout and Kopell (1998) and can be arbitrarily small. Indeed, as in Whittington, Traub, and Jefferys (1995), the I-cells can be in a parameter regime in which they would fire by themselves in the absence of excitation; the only restriction is that they not fire before the E-cells, a situation that would produce suppression of the E-cells. 
The work of Ermentrout and Kopell (1998) gave an answer to how the mechanism of Traub, Whittington, Stanford, and Jefferys (1996) could work if all the remote inputs to a local set of cells arrive with the same conduction delay. It left open the question of how the mechanism could work if a cell is to receive many inputs with a wide range of conduction delays. The role of doublets, in particular, is mysterious in such a larger network because there are now many sources of excitation, not just two. This article addresses that question and shows, surprisingly, that doublets still provide information crucial for synchronization, and that in large-parameter regimes, bursts with more than two spikes cannot support stable synchronization.

The network investigated in this article is a one-dimensional array of $N$ identical local circuits of excitatory and inhibitory cells that mimics the CA1 region of the hippocampus. There are two different architectures: a spatially ordered network and one with some disorder in the positions of the local circuits. Each local circuit receives input from a large number of others, with conduction delays proportional to the distances traveled; thus, the inputs to a local circuit come over a range of times. The connection strengths are scaled with the number of inputs, so it takes many inputs to create a response, unlike the network of Ermentrout and Kopell (1998), in which all distant inputs are lumped, and the strengths are such that a single signal from the distant circuit creates a response in the local one. The distributed network is potentially much more flexible in its response; depending on conduction times, strengths of synapses, and other parameters like the voltage threshold, the amount of disorder, and the range of connections, the number of spikes fired by an inhibitory cell in a cycle (defined by the periodic firing of the excitatory cells) can be one, two, or more, and there can be multiple solutions. The actual outcome of the network therefore depends on the stability of the solutions.

In order to perform the complicated analytical calculations associated with this extended network, we introduce a simple model of a neuron that mimics nonlinear behavior caused by ionic channels and is somewhat more complex than the standard "integrate-and-fire" neuron: a large input causes an immediate spike (and reset), and a smaller one that (with the help of previous small inputs) causes the voltage to pass some threshold eventually causes a spike, but possibly with a delay. This delay, which depends on the previous spiking history of the neuron as well as the timing of the inputs, contains the information used by the network to create the coherence. We also introduce a probability of inputs arriving at a given cell, depending on distance from that cell.

With the explicit neuronal dynamics and a lattice-like (though possibly disordered) structure, we are able to carry out computations for the probabilistic behavior. The computations lead to explicit (though complicated) formulas for the time difference between spikes of the excitatory cells at different points of the lattice as a function of their differences at the previous cycle. This is exactly the information needed to decide if synchronous be- 
havior is stable when the I-cells fire single spikes, doublets, or more spikes per cycle. That is, we do a linear stability analysis near configurations in which the spiking order and the idea of cycles are well defined. From the analysis, we can get bounds on the parameter regions in which one or more of the above synchronous solutions exists and is stable. We are able to see from this that doublets appear to provide more stability than either single spikes or a large number of spikes per cycle. Furthermore, we get the unintuitive result that a small amount of disorder in the lattice structure helps to stabilize the synchronous state. The article gives the central ideas and a broad outline of the computation; a more detailed outline is given in the appendixes. Finally, we analyze the effect of synaptic noise and find that it reduces the parameter range in which the doublet configuration has stable synchrony, although rather weakly.

The list of symbols used in this article is as follows:

$R$ the linear size of the network measured as a conduction time

$N$ the number of local circuits

$L_{i, j} \quad$ distance in time units between $i$ and $j$ circuits

$v_{l}(t) \quad$ membrane potential of the $l$ th excitatory or inhibitory cell, specified by the context

$v_{t h} \quad$ threshold potential for firing

$v_{0} \quad$ reset potential after a spike

$k_{l} \quad$ number of inputs to the $l$ th inhibitory cell after it fires its first spike until its voltage crosses $v_{l}=0$

$n_{l} \quad$ number of inputs to the $l$ th inhibitory cell after its first spike until it fires the doublet spike $\left(n_{l}>k_{l}\right)$

$a$ strength of excitatory-to-inhibitory synaptic coupling between circuits

$b \quad$ potential value of inhibitory cell after receiving the $k_{l}$ pulse

$c$ driving current in excitatory cells

$g$ amplitude of inhibition in excitatory cells

$\sigma \quad$ range of synaptic excitatory-to-inhibitory connections

$\zeta \quad$ measure of spatial disorder in positions of circuits

$\tau_{m}^{i} \quad$ membrane time constant for inhibitory cells

$\tau_{s}^{i} \quad$ inhibitory synaptic time constant between local inhibitory and excitatory cells

$\tau_{m}^{e} \quad$ membrane time constant for excitatory cells

$\tau_{s}^{e} \quad$ excitatory synaptic time constant between nonlocal excitatory and inhibitory cells

$t_{l}^{E} \quad$ spiking time of the $l$ th excitatory cell in a previous cycle

$\bar{t}_{l}^{E} \quad$ spiking time of the $l$ th excitatory cell in a next cycle

$W_{l}^{I} \quad$ delay time for firing of the $l$ th inhibitory cell

$W_{l}^{E} \quad$ delay time for firing of the $l$ th excitatory cell 


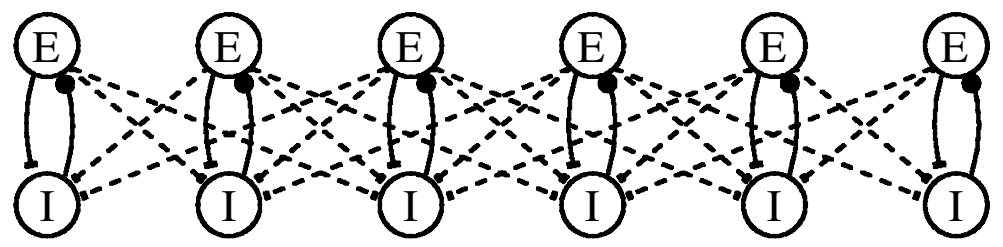

Figure 1: Network architecture. Local circuits, containing one excitatory (E) and one inhibitory (I) cell, are connected only via $\mathrm{E} \mapsto \mathrm{I}$ synapses. These connections are probabilistic in nature. The distances between neighboring circuits are constant for the ordered network. For the disordered network, these distances are slightly distorted.

\section{Network Model}

Assumptions concerning our neural network model can be decomposed into two classes: assumptions about the network architecture and assumptions about the network dynamics.

2.1 Network Architecture. The network we analyze is a one-dimensional array of linear size $R$ composed of $N$ identical local circuits, each containing one excitatory (E) and one inhibitory (I) cell with synaptic connections from E to I and I to E (see Figure 1).

For nonlocal connections, we consider only the synapses from excitatory to inhibitory cells. As in the smaller network analogue of this work (Ermentrout \& Kopell, 1998), we neglect the $\mathrm{E} \mapsto \mathrm{E}$ connections, which are sparse in the CA1 region of the hippocampus (Knowles \& Schwartzkroin, 1981). The $\mathrm{I} \mapsto \mathrm{I}$ local and nonlocal connections are treated as effectively decreasing the $\mathrm{E} \mapsto \mathrm{I}$ connections, and hence are not introduced explicitly. One simplification from the connectivity in Ermentrout and Kopell (1998) is the neglect of nonlocal $\mathrm{I} \mapsto \mathrm{E}$ connections, whose range in the CA1 area is smaller than the $\mathrm{E} \mapsto \mathrm{I}$ connections (Tamamaki \& Nojyo, 1990; Freund \& Buzsaki, 1996). Ermentrout and Kopell (1998) showed that the nonlocal $\mathrm{E} \mapsto$ I connections sufficed to produce synchrony between a pair of distant circuits (though the added connection made this synchrony more robust); we therefore investigated whether such connections would also suffice in a much larger extended network.

We treat the network architecture as probabilistic and assume that the probability $P\left(L_{i j}\right)$ of a connection between the $i$ th and $j$ th circuits separated by distance $L_{i j}$ depends on only the distance between them (translational invariance in a statistical sense). In what we describe as an ordered network, that distance is $|i-j|$; in what we shall call the disordered network, the distances are a perturbation of that quantity. In the calculations, only the times of arrival, not actual distances, are relevant so what we call "distances" 
will carry units of time. Finally, for computational reasons, we use periodic boundary conditions for our network architecture.

2.2 Network Dynamics. We denote by $v_{l}(t)$ the membrane potential of the $l$ th I-cell and the $l$ th E-cell. Its dynamics is modeled by

$$
\tau_{m}^{\alpha} \frac{d v_{l}}{d t}=v_{l} \mid+I_{l}, \quad \alpha=, i
$$

where $l=1, \ldots, N$, and $I_{l}$ represents input current. Symbols $\tau_{m}^{i}$ and $\tau_{m}^{e}$ are effective membrane time constants for the I- and E-cells. These are much smaller than the passive membrane time constant determined by the capacitance and leak current, since there are many active conductances that dominate the leak conductance (see section 5 for estimates showing that for the part of the cycle relevant to the calculation, the effective time constant of the I-cell is a fraction of a msec).

We add to the membrane dynamics represented by equation 2.1 the following property. When the potential crosses the threshold value $v_{t h}(>0)$, a cell immediately fires, and its potential is reset to $v_{0}<0$, which mimics the effect of the AHP (afterhyperpolarization) current. We assume that if the I-cell is close to recovery, a single E-spike elicits a spike from the I-cell. Thus, in the local network, the E-I circuit forms an oscillator with one spike per cycle in each cell; the I-cell has recovered by the time it receives excitation in the next cycle.

When the I-cell is partially refractory, the same input (or several inputs) to that cell may cause the potential to cross only a resting zero value; equation 2.1 then implies that the cell will also fire, but with a variable delay time that depends on the difference between the times of the excitation and the time of the last spike of that cell. This delay is a central feature of the analysis in Ermentrout and Kopell (1998) for a two-circuit network; for our more complicated case, we chose an explicit model that has this delay.

We chose the following form for the $I_{l}$ current. For an I-cell, the current is completely of synaptic origin and is given by

$$
I_{l} \Longrightarrow \sum_{k} \delta\left(t-t_{l(k)}\right) .
$$

Here the delta functions represent pulses (inputs) coming with delays from nonlocal E-cells with amplitude $a$ at times $t_{l(k)}$ from cell $l+k$ to cell $l$.

For an E-cell, the current has the form

$$
I_{l}=-g \sum_{j} e^{-\left(t-t_{l_{j}}\right) / \tau_{s}^{i}} \theta\left(t-t_{l_{j}}\right),
$$

where $c$ is a driving current (e.g., driven by a stimulus) and the second term represents inhibitory synaptic inputs with a positive amplitude $g$ coming 
from the local I-cell at times $t_{l_{j}}$ with synaptic $\mathrm{I} \mapsto \mathrm{E}$ time constant $\tau_{s}^{i}$. The function $\theta(x)$ is a standard step function defined as $\theta(x)=1$ for $x \geq 0$ and $\theta(x)=0$ for $x<0$.

We assume that for I-cells, the synaptic $\mathrm{E} \mapsto \mathrm{I}$ amplitude $a$ is smaller than both $\left|v_{0}\right|$ and $v_{t h}$. For E-cells, we assume the condition $g \gg c>v_{t h}+\left|v_{0}\right|$. The first inequality means that the local inhibition $g$ is strong enough to prevent firing (Traub, Jefferys, \& Whittington, 1999); the second implies that the Ecells fire frequently in its absence. Values $\tau_{m}^{e}, \tau_{m}^{i}, v_{0}$, and $v_{t h}$ can be different for I- and E-cells, but we do not use this explicitly.

We assume that the synaptic $\mathrm{E} \mapsto \mathrm{I}$ time course $\tau_{s}^{e}$ is not important. This assumption is consistent with experimental studies on the CA1 area (Buhl, Halasy, \& Somogi, 1994) that reveal that excitatory postsynaptic potentials to I-cells decay rapidly. As a consequence, incoming excitatory inputs to I-cells are taken as structureless (cf. equation 2.2).

As we will show, the effective membrane time constant $\tau_{m}^{i}$ for the Icells plays a role in determining parameter regimes in which the doublets are stable. The corresponding time constant $\tau_{m}^{e}$ for E-cells does not play as important a role. For some calculations, notably of $W^{E}$ defined below, we cannot get specific answers in full generality. However, we do the computation in the complementary regimes $\tau_{m}^{e} / \tau_{s}^{i} \ll 1$ and $\tau_{m}^{e} / \tau_{s}^{i} \gg 1$. Because of the large amount of active conductances that lower $\tau_{m}^{e}$, we believe $\tau_{m}^{e} / \tau_{s}^{i} \ll 1$ to be the more relevant regime. The qualitative features are shown to be the same in each, with different functional dependence of period of oscillations on $\tau_{s}^{i}$ in the two regimes. The qualitative shape of the stability diagrams does not change at all. We do not put any constraints on the relative value of $\tau_{s}^{i}$ and $\tau_{m}^{i}$.

Note that the membrane dynamics represented by equation 2.1 is piecewise-linear and slightly different from standard integrate-and-fire type models (McCullough \& Pitts, 1943; Knight, 1972; Abbott \& van Vreeswijk, 1993; Usher, Stemmler, Koch, \& Olami, 1994; Gerstner, 1995) and from the Hopfield model (Hopfield, 1984; Amit, Gutfreund, \& Sompolinsky, 1985; Hertz, Krogh, \& Palmer, 1991). Our simple model mimics some nonlinear behavior caused by ionic channels. For negative voltages, it acts as a standard leaky integrate-and-fire neuron. For positive voltages, it models the explosive depolarization generated by the opening of active voltage-gated sodium channels. Though we take the same time constants for $v<0$ and $v>0$, we note that the analysis below has the same form in the more general case in which these constants are allowed to be different. As in "type 1 neurons" (Ermentrout, 1996) for neurons in equation 2.1, there can be a significant delay between receipt of a suprathreshold signal and the response of the neuron.

In summary, in our model, the E-cells fire continuously in the absence of local inhibitory connections. With inhibition, an E-cell can fire only when the level of inhibition drops below a certain critical value. I-cells fire when they are sufficiently recovered from their last spike that the excitation (from 
whatever source) pushes them above its first $(v=0)$ threshold. Thus, if the excitation arrives long after the last spike of an I-cell, the cell fires almost immediately because it is recovered. However, the same excitation arriving shortly after a spike will not elicit another spike. We are assuming that the parameter ranges are such that an I-cell recovers well before the end of a cycle; recovery time is shorter than decay of inhibition, since the recovery time depends on the membrane time constant of the cell, which is small.

\section{Network Properties Are Revealed by Maps}

The above assumptions enable us to obtain analytical results concerning synchronous oscillations and their stability. We associate the synchronous state with the situation in which the E-cells fire simultaneously. Below we construct maps that show how the network uses the timing of spikes to accomplish synchronization.

The full cycle for E-cells is as follows. An E-cell fires and sends a pulse to its local I-cell and many nonlocal I-cells. The local I-cell fires almost immediately after receiving the pulse, since the I-cell is recovered from the last cycle. Simultaneously (or very quickly) it sends inhibition to the original E-cell, prohibiting it from further firing. In the meantime, the I-cell gets pulses from many nonlocal E-cells, but it must wait to fire, because it is not yet recovered from the first spike. We assume that the range of connectivity of the nonlocal $\mathrm{E} \mapsto \mathrm{I}$ connections (measured in time units) is significantly smaller than the inhibitory time constant $\tau_{s}^{i}$, which is about $10 \mathrm{msec}$. This implies that all the nonlocal EPSPs arrive when the local E-cell is not yet recovered from its local inhibition.

The parameter range relevant to the doublet case is one in which the combined excitation from the nonlocal E-cells is adequate to cause the Icell to fire again, after a delay we call $W^{I}$, before the E-cell recovers from inhibition and fires again. In that case, the doublet spike sends a second inhibitory pulse to the local E-cell, which will fire in the next cycle when the level of inhibition falls sufficiently. We denote the time from the second inhibitory spike to the E-cell firing by $W^{E}$. Thus for the doublet case, we have the following equations

$$
\bar{t}_{l}^{E}=W_{l}^{E}+W_{l}^{I}\left(t_{l}^{E}, t_{l(1)}, \ldots, t_{l\left(n_{l}\right)}\right)+W_{l}^{E}\left(W_{l}^{I}\right)
$$

for $l=1, \ldots, N$, where $t_{l}^{E}, \bar{t}_{l}^{E}$ are the times of spiking of the $l$ th E-cell in the previous and next cycles. $W_{l}^{I}$ is a function of $t_{l(1)}, \ldots, t_{l\left(n_{l}\right)}$, which are times of arriving pulses from nonlocal E-cells enumerated as $l(1), l(2), \ldots, l\left(n_{l}\right)$. We have $t_{l(k)}=t_{l(k)}^{E}+L_{l, l(k)}$, where $L_{l, l(k)}$ is a distance between the $l$ th and $l(k)$ th circuits. The I-cell fires at some moment between the arrival of the $n_{l}$ and $n_{l}+1$ pulses. We look for a synchronous solution-one in which $\bar{t}_{i}^{E}=\bar{t}_{j}^{E}$ provided $t_{i}^{E}=t_{j}^{E}$ for every $i$ and $j$. Equation 3.1 is the map that gives the 
timing of the E-spikes in one cycle as a function of spikes in the previous cycle. Note that the hypotheses on the extent of the excitatory connectivity is critical for well-defined cycles, which are needed to define $\bar{t}_{l}^{E}$.

In principle, one can generate multispikes in I-cells when the decay of inhibition is sufficiently slow and the range of the $\mathrm{E} \mapsto \mathrm{I}$ connectivity sufficiently large. In the case of triplets, equation 3.1 is modified to

$$
\begin{aligned}
\bar{t}_{l}^{E} \Longrightarrow & t_{l}^{E}+W_{l}^{I, 1}\left(t_{l}^{E}, t_{l(1)}, \ldots, t_{l\left(n_{l}\right)}\right)+W_{l}^{I, 2}\left(t_{l}^{E}+W_{l}^{I, 1}, t_{l\left(n_{l}+1\right)}, \ldots, t_{l\left(n_{l}+m_{l}\right)}\right) \\
& +W_{l}^{E}\left(W_{l}^{I, 1}, W_{l}^{I, 2}\right),
\end{aligned}
$$

where $W^{I, 1}$ and $W^{I, 2}$ denote the waiting time of an I-cell for the second and third spikes from the previous spike. The integer $m_{l}$ denotes the number of inputs between the occurrence of the second and third spikes. Generalization to higher-order multispikes is straightforward.

The same formalism also applies to the singlet case. In the latter, the I-cells do not receive enough excitation to overcome their partial refractoriness to reach the threshold $v=0$. Recall that the decay time of the inhibition is long enough for the I-cell to recover. Thus, an E-cell spike elicits a spike from the now-recovered I-cell, which in turn inhibits the E-cell. The formalism is as in equation 3.1, with the waiting time $W^{I}$ set to zero.

The first goal is to compute the $W^{I}$ and $W^{E}$ functions. We compute $W_{l}^{I}$ (the firing time of an I-cell started from time $t_{l}^{E}$ ) using equations 2.1 and 2.2. At time $t=t_{l}^{E}$ the potential of the I-cell is instantaneously brought to the value $v_{t h}$ by the local E-cell and equally instantaneously reset to the negative value $v_{0}$ after firing a spike. Now the I-cell begins to get excitatory pulses coming from nonlocal E-cells. The time when the I-cell crosses $v_{t h}$ is equal to $W_{l}^{I}$, which takes the form

$$
\begin{aligned}
W_{l}^{I} t_{l\left(n_{l}\right)}-t_{l}^{E}-\tau_{m}^{i} \ln [ & \frac{b}{v_{t h}} \exp \left[\left(t_{l\left(n_{l}\right)}-t_{l}^{E}-\delta t_{l}\right) / \tau_{m}^{i}\right] \\
& \left.+\frac{a}{v_{t h}} \sum_{i=k_{l}}^{n_{l}} \exp \left[\left(t_{l\left(n_{l}\right)}-t_{l(i)}\right) / \tau_{m}^{i}\right]\right],
\end{aligned}
$$

where $k_{l}$ is the number of inputs needed for the I-cell to cross $v_{l}=0$, and $\delta t_{l}$ is the time at which this happens. The $k_{l}$ th pulse takes the voltage from a negative to some positive value $b$ satisfying $0<b<a$. The I-cell fires after receiving $n_{l}$ excitatory inputs. The details of the derivation of equation 3.3 are presented in appendix A.

Computation of $W_{l}^{E}$ using equations 2.1 and 2.3 is presented in appendix B. Here we briefly sketch (Ermentrout \& Kopell, 1998) how to derive an approximate formula for $W_{l}^{E}$. In the limit of fast membrane dynamics of the E-cell relative to the synaptic $\mathrm{I} \mapsto \mathrm{E}$ time course, that is, when $\tau_{m}^{e} / \tau_{s}^{i} \ll 1$, 
we can set the left-hand side of equation 2.1 with $\alpha=e$ to zero. The inhibitory current felt by an E-cell decreases with time as $g \exp \left(-t / \tau_{s}^{i}\right)$. In the case of doublets, one has two inhibitory spikes separated by time $W_{l}^{I}$; the total inhibition is $g\left[\exp \left(-t / \tau_{s}^{i}\right)+\exp \left(-\left(t-W^{I}\right) / \tau_{s}^{i}\right)\right]$. Similarly, for triplets one has three exponential terms. Our $W_{l}^{E}$ is the time interval from the occurrence of the second inhibitory spike to the local E-cell when the total inhibition drops below a certain critical value. That value is the one for which the total current $I_{l}$ in equation 2.3 becomes positive. After the current $I_{l}$ becomes positive, equation 2.1 implies that the membrane potential starts to grow, reaching the threshold very quickly at a time of the order of $\tau_{m}^{e}$. In that limit $W_{l}^{E}$ takes the form

$$
W_{l}^{E}=\tau_{s}^{i} \ln \left[\frac{g}{c}\left(1+e^{-W_{l}^{I} / \tau_{s}^{i}}\right)\right]
$$

at that time, the E-cell fires in the next cycle. In the opposite limit, $\tau_{m}^{e} / \tau_{s}^{i} \gg 1$, $W_{l}^{E}$ is of the order of the membrane time constant $\tau_{m}^{e}$ with a similar functional dependence (cf. appendix B).

In the disordered network, the arriving times of pulses from E-cells to the $l$ th I-cell form an irregular pattern. Therefore, we must introduce averaging over those times. We assume that the inputs are independent of one another and their temporal pattern depends only on initial conditions $\left(t_{l}^{E}\right)$ and the architecture of the network $\left\{L_{l, l(k)}\right\}$. We average equations 3.1 and 3.2 over the network architecture in which $l(k) \Longrightarrow+k(\bmod N)$; this corresponds to the assumption about periodic boundary conditions. Since arrival times do not depend on each other, our probability distribution $\rho$ of $\left\{L_{l, l+k}\right\}$ is of the form

$$
\rho\left(L_{l, l+1}, \ldots, L_{l, l+N-1}\right)=\prod_{k=1}^{N-1} P\left(L_{l, l+k}\right) .
$$

We choose the probability $P\left(L_{l, l+k}\right)$ of input arriving from $(l+k)$ th circuit to $l$ th circuit at time $L_{l, l+k}$ to be

$$
P\left(L_{l, l+k}\right) \sim \frac{1}{2}\left[\delta\left(l, l+k-\frac{k R}{N}-\zeta\right) \delta\left(l, l+k-\frac{k R}{N}+\zeta\right)\right)^{-L_{l, l+k} / \sigma},
$$

where $R$ is a linear size of the system, $\zeta$ is a measure of spatial disorder in positions of neurons and is the same for every pair of neurons, and $\sigma$ represents the range of synaptic connections between neurons. $\sigma$ is chosen so that if the E-cells are synchronous, all the excitation from the nonlocal E-cells to a local circuit comes during the recovery of a local E-cell from its local inhibition. Note that $\rho$ does not depend on the position of the $l$ th circuit, which is a consequence of the assumption about the translational invariance. The probability $P\left(L_{l, l+k}\right)$ is a product of two terms: the one with 
the delta functions represents the probability of arrival of an input at certain time, and the one with the exponential decay represents the probability of synaptic connection between circuits. The latter factor mimics the locality of connections. When $\sigma=\infty$, we have a network with global $\mathrm{E} \mapsto$ I connections.

The advantage of formula 3.6 lies in the fact that it captures different types of network architecture, yet is very simple and suitable for analytical investigations. When $\zeta=0$, one has an ordered network with regularly arriving pulses separated by time $R / N$. The case with $\zeta \rightleftharpoons 0$ corresponds to a disordered network in which the positions of the neurons are irregular or alternatively in which the positions of the neurons are regular but the lengths of axonal connections between them are irregular. In such a network, pulses come in at times that are random but symmetrically distributed around $k R / N$ for every $k$. In principle, the range of variability of $\zeta$ can be from zero to $R / 2 N$. However, in order to make our calculations easier, we adopted the weak disorder limit corresponding to $\zeta N / R \ll 1$. In this limit, the moments of distribution 3.6 are the same as those of a gaussian distribution for arriving times (cf. appendix C). Since the results of this article depend only on the moments, this implies that the results are equivalent for the two distributions.

For the sake of convenience we define $\Delta_{l-1} \equiv\left\langle t_{l}^{E}\right\rangle-\left\langle t_{1}^{E}\right\rangle$, where the symbol $\langle\ldots\rangle$ denotes averaging with respect to the probability distribution 3.5 and 3.6 , that is,

$$
\langle H\rangle=\int_{0}^{\infty} d L_{l, l+1} \ldots \int_{0}^{\infty} d L_{l, l+N-1} \rho\left(L_{l, l+1}, \ldots, L_{l, l+N-1}\right) \cdot H,
$$

for any function $H$ depending on $\left\{L_{l, l+k}\right\}$.

The next step is to rewrite equations 3.1 and 3.2 in terms of the $\{\Delta\}$. This gives us $(N-1)$ equations for $\bar{\Delta}_{l} \equiv\left\langle\bar{t}_{l+1}^{E}\right\rangle-\left\langle\bar{t}_{1}^{E}\right\rangle$, which constitute the multidimensional map

$$
\bar{\Delta}_{l}=f_{l}\left(\Delta_{1}, \ldots, \Delta_{N-1}\right) .
$$

(Recall that the overbar denotes a quantity associated with the next cycle.) In the case of the doublet configuration, the nonlinear function $f=$ $\left(f_{1}, f_{2}, \ldots, f_{N-1}\right)$ is given by

$$
\left.f_{l}\left(\Delta_{1}, \ldots, \Delta_{N-1}\right)=\bar{t}_{l+1}^{E}(\{\Delta\})\right\rangle-\left\langle\bar{t}_{1}^{E}(\{\Delta\})\right\rangle,
$$

where

$$
\left\langle\bar{t}_{l}^{E}(\{\Delta\})\right\rangle=\left|t_{l}^{E}\right\rangle+\left\langle W_{l}^{I}\right\rangle+\left\langle W_{l}^{E}\right\rangle .
$$

This means that the average of the next E-cell firing is a function only of the last E-cell average firing time, with no previous history dependence. In 
equation 3.10, $\left.\left.W_{l}^{I}\left(L_{l, l(1)}, \ldots, L_{l, l\left(n_{l}\right)}\right) ; \Delta\right\}\right)$ and $W_{l}^{E}\left(L_{l, l l(1)}, \ldots, L_{l, l\left(n_{l}\right)} ;\{\Delta\}\right)$ are now functions of $\left\{L_{l, l(k)}\right\}$ and $\{\Delta\}$ instead of the arrival times $\left\{t_{l(k)}\right\}$. Formulas for $\left\langle W_{l}^{I}\right\rangle$ and $\left\langle W_{l}^{E}\right\rangle$ are complicated; their sum has the form

$$
\begin{aligned}
& \left\langle W_{l}^{I}\right\rangle+\left\langle W_{l}^{E}\right\rangle=\frac{\tau_{s}^{i}}{2^{n_{l}} \cosh ^{n_{l}}(\zeta / \sigma)} \sum_{\alpha_{1}, \ldots, \alpha_{n_{l}}=1}^{2} \exp \left(-\sum_{j=1}^{n_{l}}(-1)^{\alpha_{j}} \zeta / \sigma\right) \\
& \times \ln \left[\frac { g } { c } \left(+\exp \left[W_{l}^{I} \frac{C}{L}+(-1)^{\alpha_{1}} \zeta, \ldots, \frac{n_{l} R}{N}\right.\right.\right.
\end{aligned}
$$

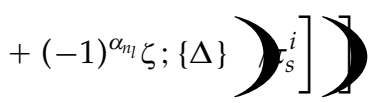

with $W_{l}^{I}\left(\frac{R}{N}+(-1)^{\alpha_{1}} \zeta, \ldots, \frac{n_{l} R}{N}+(-1)^{\alpha_{n_{l}} \zeta} ;\{\Delta\}\right)$ given by equation 3.3 . The derivation of equation 3.11, including details of how to compute average values of $k_{l}$ and $n_{l}$, which we denote as $k$ and $n$, respectively, is given in appendix $D$. We are interested in the existence and stability of a synchronous state, which corresponds to the fixed point $\bar{\Delta}_{l}=\Delta_{l}$ with $\Delta_{l}=$, for every l. For the triplet case, the function $f$ can be obtained explicitly as well but is more complex.

In principle, the I-cells may produce many spikes in a cycle, provided the size $R$ of the system is sufficiently large. Some of these spiking configurations may be stable; some may not (see below). In the case of multistability, the actual configuration depends on the values of parameters used in the system. Especially critical is the interplay between the synaptic strength $a$ and the linear size of the system $R$. The synaptic strength determines how many inputs from E-cells are needed to trigger firing of an I-cell. To have only doublets, one has to have a sufficient number of inputs to trigger the second spike in I-cell, that is, $n<N$, and insufficient to trigger a third spike, which means $(n+k)>N$. Using the formulas for $k_{l}$ and $n_{l}$ in appendix $\mathrm{D}$, we can use these inequalities to get bounds on the synaptic amplitude $a$ such that the system has only doublets. When $R / N \tau_{m}^{i} \gg 1$ and $0<\zeta / \tau_{m}^{i} \simeq 1$, which imply weak disorder, these bounds may be written as

$$
\begin{aligned}
\frac{\cosh \frac{\zeta}{\sigma}}{\cosh \left(\frac{\zeta}{\sigma}-\frac{\zeta}{\tau_{m}^{i}}\right)} e^{-R / \tau_{m}^{i}} & <\frac{a b}{v_{t h}\left|v_{0}\right|} \\
& < \\
& \left(\frac{\cosh \left(\frac{\zeta}{\sigma}+\frac{\zeta}{\tau_{m}^{i}}\right)}{\cosh \left(\frac{\zeta}{\sigma}-\frac{\zeta}{\tau_{m}^{l}}\right)}\right)^{1 / 2} e^{-R / 2 \tau_{m}^{i}} .
\end{aligned}
$$

For larger $a$ it is possible to have both doublets and higher-order multispikes. In that case, the system would choose a stable configuration (see section 5). For stronger disorder (but still weak) when $R / N \tau_{m}^{i} \gg \zeta / \tau_{m}^{i} \gg 1$, the formula is slightly more complex, but the conclusion about $a$ is still valid. 
We check the stability of synchronous solutions using the $(N-1)$ by $(N-1)$ stability matrix $A$ defined as $A_{l, m}=\partial \bar{\Delta}_{l} /\left.\partial \Delta_{m}\right|_{\Delta=\theta}$. The synchronized fixed point is stable when the modulus of all eigenvalues $\lambda_{l}$ of $A$ is smaller than unity. Invoking the Gershgorin theorem (Horn \& Johnson, 1985), one can determine an upper limit of $\left|\lambda_{l}\right|$ as $\sum_{m=1}^{N-1}\left|A_{l, m}\right|$. If this upper limit is smaller than unity, we obtain a sufficient condition for stability that allows us to compute the stability diagrams presented in Figure 2. In these figures, we plot the region in parameter space in which the sufficient condition holds. We have chosen to vary $R / N \tau_{m}^{i}$ and $\tau_{m}^{i} / \tau_{s}^{i}$, since they emerge naturally as suitable coordinates; the other parameters are fixed. Details of this computation are included in appendix E.

For the singlet case we find that the modulus of each eigenvalue of $A$ is one; that is, the synchronized oscillations with singlets are marginally stable. In the case of doublets, we obtain stability regions for both the ordered and the disordered network. A crucial factor for this stability is the presence of delay time functions $\left\langle W^{I}\right\rangle$ in equation 3.10. To make the calculated stability diagrams plausible, we now show that diagrams such as the ones depicted in Figure 2 are to be expected for solutions with doublets. Both figures show that for sufficiently small values of $R / N \tau_{m}^{i}$, the doublets are unstable. To get some insight into this behavior, notice that in order for an I-cell to fire, it must receive a sufficient number of inputs to cross the zero value of its potential. Therefore, the size of the system $R$ must be sufficient to produce enough inputs. The value of $R / N$ plays the same role in this network as does the delay time $\delta$ in the network for two circuits (Ermentrout \& Kopell, 1998); in the latter network, stability requires $\delta$ to be above some minimum magnitude, provided the coupling is not too strong. Similarly, when the effective membrane time constant $\tau_{m}^{i}$ (from $v_{0}$ to zero) is too large relative to the number of inputs, then the I-cell will not fire; hence doublets cannot exist.

Figure 2 shows that ordered and disordered networks differ with respect to stability for a large $\tau_{m}^{i} / \tau_{s}^{i}$ ratio. This can be made plausible, again invoking existence arguments. If the synaptic time constant $\tau_{s}^{i}$ of an I-cell is too small, the inhibition felt by an E-cell falls off faster than arrival of inputs to produce a second spike in the I-cell. This means that to get doublets, an inequality $\left.\tau_{s}^{i}\right\rangle$ $\left\langle W^{I}\right\rangle$ must be satisfied. For the ordered network, we have $\left\langle W^{I}\right\rangle \sim \tau_{m}^{i}$, since the time between doublets of the I-cell is governed by its effective membrane time constant $\tau_{m}^{i}$ (cf. appendix D). Therefore we find that ratio $\tau_{m}^{i} / \tau_{s}^{i}$ must have an upper bound, because $\left\langle W^{I}\right\rangle$ is bounded from below and positive (see Figure 2A). For the disordered network, our $\left\langle W^{I}\right\rangle$ depends additionally on the disorder parameter $\zeta$. This dependence creates a dramatic change, since now $\left\langle W^{I}\right\rangle$ can assume arbitrarily small values for sufficiently large $\zeta$. That, in turn, implies that $\tau_{m}^{i} / \tau_{s}^{i}$ does not have an upper bound because $\tau_{m}^{i} / \tau_{s}^{i} \sim 1 /\left\langle W^{I}\right\rangle$.

Using the stability matrix $A$, we can calculate the critical value of $\left(R / N \tau_{m}^{i}\right)_{c r}$; above that value the sufficient condition for stability is satisfied 

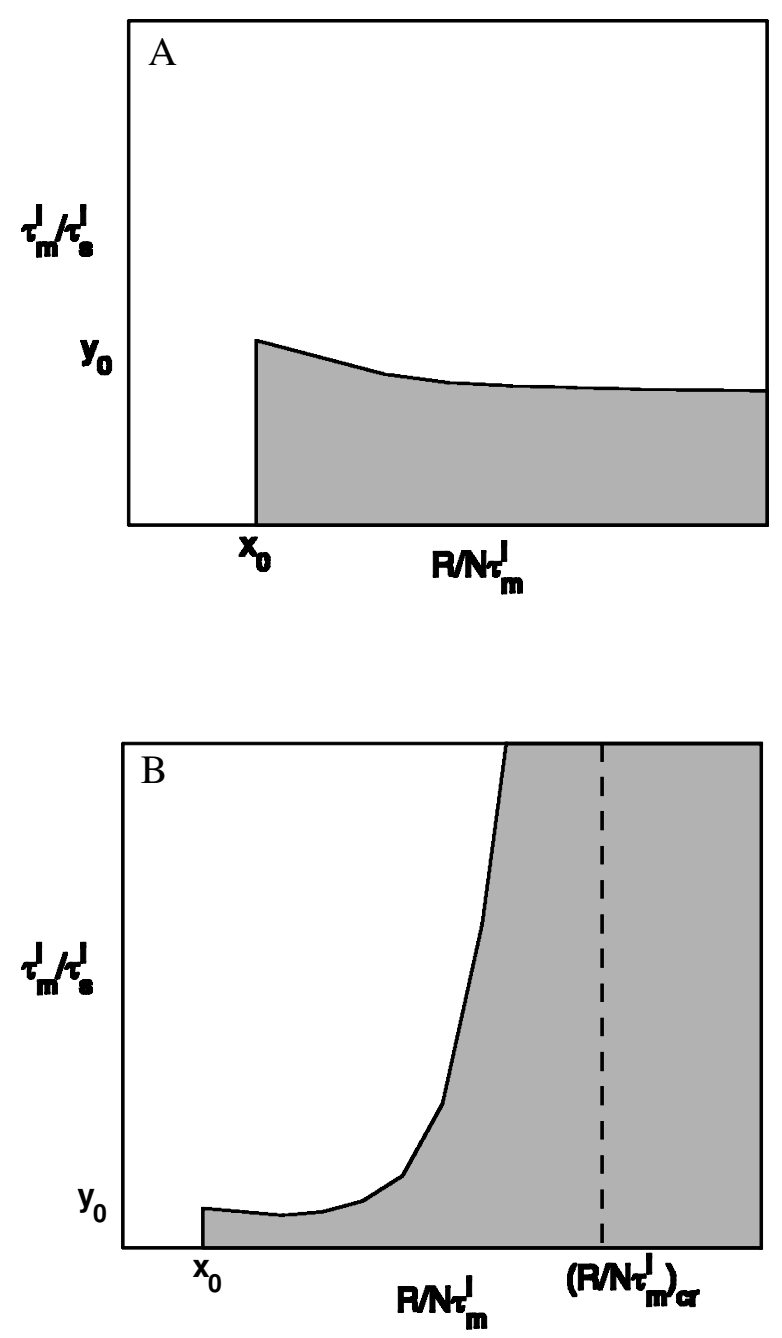

Figure 2: Stability diagrams for the doublet configuration in the regime $\tau_{m}^{e} / \tau_{s}^{i} \ll$ 1. The shadowed area indicates the stability region. (A) Ordered network. Values $x_{0}$ and $y_{0}$ present on the axes are given by equations E.9 and E.10 in sppendix E, respectively. There is stability only for $\tau_{m}^{i} / \tau_{s}^{i} \ll 1$ and for $R / N \tau_{m}^{i}>x_{0}$. For values $b=a / 2$ and $\left|v_{0}\right| / a \Longrightarrow$, one finds $x_{0} \approx 2$. (B) Disordered network. Note the appearance of the vertical asymptote and the increase in the stability region. Values $x_{0}$ and $y_{0}$ are of the same order of magnitude as for the ordered network. In the complementary regime (i.e., when $\tau_{m}^{e} / \tau_{s}^{i} \gg 1$ ), the shapes of the diagrams remain the same with a substitution $\tau_{s}^{i} \mapsto \tau_{m}^{e}$ in coordinates. 
for arbitrary values of $\tau_{m}^{i} / \tau_{s}^{i}$ (see Figure $2 \mathrm{~B}$ ). In the limit $R / N \tau_{m}^{i} \gg \zeta / \tau_{m}^{i} \gg 1$, this critical value takes the form

$$
\left(R / N \tau_{m}^{i}\right)_{c r} \approx \frac{R}{N \sigma}+\frac{R}{N \zeta} \ln \left[\frac{8\left|v_{0}\right|}{a} \cosh ^{2} \frac{\zeta}{\sigma}\right]
$$

which is the point where a vertical asymptote crosses the horizontal axis in Figure 2B. Notice that when $\zeta=0$, namely, for the ordered network, this asymptote shifts to infinity, reducing the stability region. Also note that the stability region grows with an increasing range of connections $\sigma$ and synaptic strength $a$. Details for how to compute $\left(R / N \tau_{m}^{i}\right)_{c r}$ are given in appendix E.

For the triplet case in the ordered network, we find that the upper limit of $\left|\lambda_{l}\right|$ is always greater than unity (computation not given). This suggests that the triplet configuration in a such network is unstable. On the contrary, in the disordered network we find that synchronized oscillations with triplets are stable for all $\tau_{m}^{i} / \tau_{s}^{i}$ provided $R / N \tau_{m}^{i}$ ratio is sufficiently large, that is, $R / N \tau_{m}^{i} \gg 1$, although we are unable to determine the exact values analytically.

We see from the above that spatial disorder in a network helps to stabilize the synchronized oscillations. So does increasing the range of synaptic connections (see equation 3.13). Based on comparison between doublets and triplets, we conjecture that higher-order multispikes are less stable than doublets, if they exist at all.

The analysis also allows us to determine the period $T$ of oscillations, which is equal to $\left\langle W^{I}\right\rangle+\left\langle W^{E}\right\rangle$ (see equation 3.10). In the ordered network, with the doublet configuration, a formula is relatively simple. In the limit $\tau_{m}^{e} / \tau_{s}^{i} \ll 1$ we obtain

$$
\begin{aligned}
T=\tau_{s}^{i} \ln \left(\frac{g}{c}[1+\right. & \frac{\left[\left(v_{t h}-a\right)\left(1-e^{-R / N \tau_{m}^{i}}\right)+a\right]}{a\left[b+(a-b) e^{-R / N \tau_{m}^{i}}\right]} \\
& \left.\left.\times\left[a+\left|v_{0}\right|\left(1-e^{-R / N \tau_{m}^{i}}\right)\right]\right)^{\tau_{m}^{i} / \tau_{s}^{i}}\right] .
\end{aligned}
$$

Note that the period of oscillations of the whole network is larger than the period of oscillations of an isolated local circuit, $\tau_{s}^{i} \ln (g / c)$, but both are of the same order of magnitude. In the opposite limit, $\tau_{m}^{e} / \tau_{s}^{i} \gg 1$, one should substitute $\tau_{m}^{e}$ for $\tau_{s}^{i}$ and $g\left(1+v_{t h} / c\right) \tau_{s}^{i} / \tau_{m}^{e}$ for $g$.

The period grows with increasing size of the system, saturating for $R \mapsto$ $\infty$ (for fixed $N$ ). This means that the frequency of oscillations is bounded from below. The dependence on the membrane time constant $\tau_{m}^{i}$ is essentially the same as on $R$. $T$ decreases with the increasing strength of $E \mapsto I$ 
synapses, which corresponds to increasing the amplitude $a$. The same conclusions are valid in the case of the disordered network with the doublet solution, but the expression for the period is more complex with similar logarithmic terms.

In the regime $\tau_{m}^{e} / \tau_{s}^{i} \ll 1$, we obtain the following expressions for the period of oscillations. For the ordered network, in the limits $\tau_{m}^{i} / \tau_{s}^{i} \ll 1$ and $R / N \tau_{m}^{i} \gg 1$, that is, in the parameter range where there is a stable doublet configuration, our period is governed only by the inhibitory synaptic time course $\tau_{s}^{i}$ (Whittington et al., 1995; Jefferys, Traub, \& Whittington, 1996; Traub, Whittington, Colling, Buzsaki, \& Jefferys, 1996) and reads $T \approx \tau_{s}^{i} \ln (2 g / \mathcal{c})$. (Proportionality between the period and $\tau_{s}^{i}$ has also been obtained before in a network of inhibitory neurons; Chow, White, Ritt, \& Kopell, 1998.) In the above limits, we also obtain a very simple formula for the ratio of the averaged timing between doublets $\left\langle W^{I}\right\rangle$ to the period$\left\langle W^{I}\right\rangle / T \approx\left(\tau_{m}^{i} / \tau_{s}^{i}\right) \ln \left(v_{t h}\left|v_{0}\right| / a b\right) / \ln (2 g / c)$-which depends logarithmically on the synaptic nonlocal $(\mathrm{E} \mapsto \mathrm{I})$ strength. In the same limit for the disordered network with additional constraint $\zeta / \tau_{m}^{i} \preceq 1$, we obtain the period

$$
T \approx \tau_{s}^{i} \ln \left(\frac{\delta}{2}\right) \frac{\zeta}{2} \tanh \frac{\zeta}{\sigma}
$$

This implies that spatial disorder reduces the period, and in addition, the longer the range of connections $\sigma$, the greater is the period.

In the complementary regime $\tau_{m}^{e} / \tau_{s}^{i} \gg 1$, we obtain the following expression for the period:

$$
\left.T \approx \tau_{m}^{e} \ln \frac{\mathcal{\delta} \tau_{s}^{i}}{\tau_{m}^{e}}\left[1+\frac{v_{t h}}{c}\right]\right) \frac{\zeta}{2} \tanh \frac{\zeta}{\sigma} .
$$

Note that now the period depends more weakly (i.e., logarithmically) on the synaptic time constant $\tau_{s}^{i}$.

The triplet case occurs only in the disordered network. Also here, an analytical expression for $T$ can be obtained, and again we find that the leading dependence is logarithmic, although a formula is more complicated than for doublets. Nevertheless, the qualitative dependences on $R, \tau_{s}^{i}, \tau_{m}^{i}, a$ and the disorder remain valid here.

\section{Inclusion of Synaptic Noise}

In this section we estimate the effect of a synaptic noise on the stability of the doublet configuration in the regime $\tau_{m}^{e} / \tau_{s}^{i} \ll 1$. To simplify considerations we will consider the network with $N=2$ local circuits with the same type of connections as before.

For $N=2$ circuits, the assumption about the smallness of excitatory $\mathrm{E} \mapsto \mathrm{I}$ synaptic strength $a$ is removed; in this case, a single nonlocal excitatory input 
must trigger the firing of local I-cell. We assume, however, that $a<v_{t h}$, so that an I-cell has a delay between receipt of an input and firing.

We can make use of equations 3.3 and 3.4 derived before for $W^{I}$ and $W^{E}$ functions. The formula for $W^{I}$ will be modified because now there is only one strong source of excitation instead of many weak sources. Thus, the sum in the logarithmic term in equation 3.3 now disappears. We note that the time $\delta t_{l}$ when the membrane potential crosses zero is exactly the moment when nonlocal excitatory pulse arrives, $\delta t_{l}=t_{2}^{E}-t_{1}^{E}+R$, where $R$ is now the conduction delay time between the circuits. The formula for the value of the membrane potential $b$ right after the reception of an input can also be substituted in equation 3.3. This value now is $b=v_{0} \exp \left(-\delta t_{l} / \tau_{m}^{i}\right)+a$. Taking all these changes into account, we obtain for the first circuit

$$
W_{1}^{I} \Longrightarrow t_{2}^{E}+R-t_{1}^{E}+\tau_{m}^{i} \ln \left(\frac{v_{t h}}{v_{0} e^{-\left(t_{2}^{E}+R-t_{1}^{E}\right) / \tau_{m}^{i}}+a}\right) .
$$

A similar formula holds for the second circuit with the substitution $t_{1}^{E} \leftrightarrow t_{2}^{E}$. The equation for $W_{1,2}^{E}$ remains the same as equation 3.4

In the presence of synaptic noise, synaptic strengths $a$ and $g$ will fluctuate rapidly around their average values, reducing the precision in values of $W^{I}$ and $W^{E}$. To estimate the effect of synaptic noise, we will treat $a$ and $g$ as random variables.

Repeating the steps from section 3 we have

$$
\bar{\Delta} \Longrightarrow \Delta+\tau_{s}^{i} \ln \left(\frac{K-\Delta)}{k(\Delta)}\right)
$$

where now $\Delta \equiv t_{2}^{E}-t_{1}^{E}$ and $\bar{\Delta} \equiv \bar{t}_{2}^{E}-\bar{t}_{1}^{E}$, and

$$
h(\Delta)=\left(v_{0} e^{-(R+\Delta) / \tau_{m}^{i}}+a\right)^{\tau_{m}^{i} / \tau_{s}^{i}}+\left(e^{(R+\Delta) / \tau_{m}^{i}} v_{t h}\right)^{\tau_{m}^{i} / \tau_{s}^{i}} .
$$

Notice that synaptic $\mathrm{I} \mapsto$ E strength $g$ cancels out. Averaging the above equation over different possible values of $a$ with a simple probability distribution (cf. appendix C) $P(a)_{\text {noise }}=\frac{1}{2}\left[\delta\left(a-a_{0}-\delta a\right)+\delta\left(a-a_{0}+\delta a\right)\right]$, where $\delta a$ is a measure of synaptic noise, and solving the stability inequality $|\partial \bar{\Delta} / \partial \Delta|_{\Delta=\theta} \mid<1$ in terms of $R$, in the limit $\delta a / a_{0} \ll 1$ we obtain, after some tedious algebra,

$$
R / \tau_{m}^{i}>\ln \left(\frac{2\left|v_{0}\right|}{a_{0}}\left[1+r\left(\frac{a}{a}\right)^{2}\right]\right)
$$

with

$$
r=\frac{2\left[\left(4\left|v_{0}\right| v_{t h}\right)^{\tau_{m}^{i} / \tau_{s}^{i}}+a_{0}^{2 \tau_{m}^{i} / \tau_{s}}\left(1+\tau_{m}^{i} / \tau_{s}^{i}\right)\right]}{\left(4\left|v_{0}\right| v_{t h}\right) \tau_{m}^{i} / \tau_{s}^{i}+a_{0}^{2} \tau_{m}^{i} / \tau_{s}} .
$$


When the synaptic noise is absent $(\delta a=0)$, the right-hand side of equation 4.4 is smaller than if $\delta a>0$. Hence, inclusion of synaptic noise decreases the parameter regime in which there is stability. However, the effect of noise is rather weak, since dependence in equation 4.4 is logarithmic.

Finally, let us note that since $g$ dropped out from equations 4.4 and 4.5, synaptic noise in a local $\mathrm{I} \mapsto \mathrm{E}$ inhibitory synapse is irrelevant. This is the consequence of the fact that we assumed slow $\mathrm{I} \mapsto$ E inhibition, that is, $\tau_{m}^{e} / \tau_{s}^{i} \ll 1$. When inhibition is slow, fast synaptic fluctuations do not have a great influence on synaptic transmission, since the synapse has time to average over fluctuations. This is not the case for $\mathrm{E} \mapsto \mathrm{I}$ nonlocal synaptic connections, since in that case excitation is fast (Buhl et al., 1994) and the system lacks the time to average. Therefore, synaptic fluctuations in the latter case are important.

\section{Discussion}

The literature on synchronization is very large; some related papers using the properties of inhibition are Lytton and Sejnowski (1991), Wang and Rinzel (1992, 1993), Friesen (1994), van Vreeswijk, Abbott, and Ermentrout (1994), Bush and Sejnowski (1996), Gerstner, van Hemmen, \& Cowan (1996), Wang and Buzsaki (1996), Terman, Kopell, and Bose (1998), Terman and Rubin (1998), White, Chow, Ritt, Soto, \& Kopell (1998), and Chow et al. (1998). In most articles on synchronization, conduction delays do not play a part. One that explicitly addresses that question is Konig and Schillen (1991), which gives simulations of a firing-rate model showing that synchronization could be accomplished with delays up to roughly a third of the period. It differs in two respects from our article. First, we consider the spiking neurons type model, in which the timing between single spikes is very important; in firing-rate models, the fine temporal structure is lost. Second, our synchronous state is stable even for infinite-range delays, that is, for $\sigma \mapsto \infty$ (see equation 3.13 and Figure 2B); if there is heterogeneity in the driving current, the range could be reduced (Ermentrout \& Kopell 1998).

Crook, Ermentrout, Vanier, and Bower (1997) considers temporal delays caused by axons in a continuous model of excitatory oscillators. They found that the synchronous state is stable provided delays are not too large. These authors do not consider the effect of inhibition. Other articles, like Campbell and Wang (1998) and Ernst, Pawelzik, and Geisel (1995) deal with pulsecoupled relaxation oscillators. The first of these uses excitation with homogeneous global inhibition. The second uses either excitatory or inhibitory homogeneous connections, but not both, as a mechanism for generation of long-range synchronization. Neither of these articles takes into account the time structure of inhibition. Thus, they are quite different in spirit from the models of Whittington et al. (1995), Traub, Whittington, Stanford, and Jefferys (1996) and Ermentrout and Kopell (1998), which deal with synchronization of excitatory and inhibitory cells that spike once or twice per cycle, 
and make central use of the time course of the inhibition. Another recent related article is Traub, Whittington, Stanford, and Jefferys (1999), which includes a more detailed model than the one in Traub, Whittington, Stanford, and Jefferys (1996) and summarizes a body of work on gamma and beta rhythms. (See also Traub, Jefferys, \& Whittington, 1999.)

This article extends the work of Ermentrout and Kopell (1998) on gamma oscillations in a smaller network. We have analyzed the mechanism for producing long-range synchrony in a distributed network of spiking neurons, in which each interneuron receives a large number of inputs arriving at different times. We showed that even with many inputs, a firing configuration in which interneurons produce doublets yields stable synchronization over a larger-parameter range than other configurations. These doublets have been seen in large-scale simulations, as well as in vitro studies (Traub, Whittington, Stanford, \& Jefferys, 1996; Traub, Whittington, Buhl, Jefferys, \& Faulkner, 1999), and might provide additional support for a temporal coding hypothesis (Rieke, Warland, de Ruyter van Steveninck, \& Bialek, 1997). We note that (as in Tsodyks, Mitkov, \& Sompolinsky, 1993, van Vreeswijk et al., 1994, and Gerstner et al., 1996), our calculations deal only with linear stability, and hence do not give information about global stability.

In Ermentrout and Kopell (1998), the synchronization mechanism depends on the properties of the waiting time function called $W^{I}$ in this article and $T_{I}$ in Ermentrout and Kopell (1998). The analysis in that article was valid for all functions $T_{I}$ that have the qualitative property that they decay in time. That is, the longer the time from the spike of an I-cell until when it receives further excitation, the shorter the wait until it then fires. This property was shown to hold for a class of biophysical models reduced from those of Traub and Miles (Ermentrout \& Kopell, 1998; Traub \& Miles, 1991). The analysis in this article uses a particular form for the dynamics (see equation 2.1), a one-dimensional system having two thresholds. This form was chosen as the simplest equation for which the waiting time function $T_{I}$ has a form similar in shape to that computed from the reduced model of Traub and Miles (1991). This was used in the computation of equation 3.3.

The essential role in our mechanism is played by local inhibitory neurons that filter inputs coming from nonlocal excitatory cells to a local Ecell, inhibiting it from firing by producing appropriately timed multispikes. Especially in the regime $\tau_{m}^{e} / \tau_{s}^{i} \ll 1$, this inhibition governs the period of oscillations of excitatory cells, as well as stabilizes the synchronous state. For the ordered network (see Figure 2A) this inhibition must be slower than the effective membrane time constant of inhibitory cells in order to preserve stability. In the case of the disordered network (see Figure 2B) this inhibition does not have to be slow for sufficiently large $R / N \tau_{m}^{i}$. In the complementary regime, $\tau_{m}^{e} / \tau_{s}^{i} \gg 1$, the time course of the inhibition does not play any role in the stability.

Our stability diagrams and the period of oscillations scale with factor $R / N$, the average time needed for a pulse to travel between neighboring 
circuits. This scaling is a direct consequence of equation 3.6 and indicates that the stability diagrams, as well as the period, do not depend on the network size $R$, provided the density of circuits $R / N$ is fixed. The stability diagrams indicate that below a minimal value $x_{0}$ of $R / N \tau_{m}^{i}$, the doublet configuration is not stable. From equation E.9 (in appendix E), using as parameter values $b \Longrightarrow a / 2$, and $v_{0} / a \Longrightarrow$, we get $x_{0} \approx 2$, with $x_{0}$ only weakly dependent on $a, b$ and $v_{0}$. This gives a minimal size for the distance $R / N$ between the local circuits: $R / N>x_{0} \tau_{m}^{i}$. If the effective time constant $\tau_{m}^{i}$ is very small (from conductances in Traub, Whittington, Stanford, \& Jefferys, 1999, we estimate $\tau_{m}^{i}$ to be a fraction of a msec), the local circuits can be taken to be very close.

This estimate is made as follows: the active conductances include an excitatory metabotropic conductance, AHP conductance and synaptic conductances from local interactions (Traub, Whittington, Stanford, \& Jefferys, 1999). For interneurons, the input conductance is about $10 \mathrm{nS}$. After the first spike, there is a large, fast AHP. At the peak of the fast AHP, the input conductance is at least 500 to $1000 \mathrm{nS}$ (Traub \& Miles, 1995). That means that after the first action potential, the input conductance increases by at least a factor of 50, which implies that the time constant goes down by such a factor. An estimate of the baseline time constant in Traub and Miles (1995) is $37.5 \mathrm{msec}$; after the first action potential and before the doublet, the time constant would then be less than a msec. This takes into account only the AHP current. If one includes also the metabotropic glutamate conductances activated by the tetanic stimulation and the $\mathrm{GABA}_{\mathrm{A}}$ conductances from the other interneurons, as documented in Whittington, Stanford, Colling, Jefferys, \& Traub (1997), one has an effective time constant that is considerably lower. We note that all the computations done in this article concern the time between the first and second spikes of a doublet (or the third, in the triplet configuration). Thus we are using an effective time constant that reflects only the small part of the oscillation cycle.

Another property of equation 3.6 is that the disorder parameter $\zeta$ is the same for every pair of circuits. This is the consequence of assumed translational invariance in the system and corresponds to the situation in which disorder is not too strong. We showed that for weak spatial disorder, the results of this article are identical if equation 3.6 is replaced by a gaussian distribution. In the limit of very strong disorder, however, one should not expect the assumption about translational invariance to be correct. In this case, the proper approach would require taking $\zeta$ as a space-dependent variable, that is, different for every pair of circuits.

Our analysis concerns perfect (probabilistic) synchrony, in which every cell (excitatory and inhibitory) participates in the synchronization process. For such a complete synchrony, we find that weak disorder $(\zeta N / R \ll 1)$ enlarges the stability region of the synchronous state in parameter space in comparison to the ordered network. Our argument probably does not work for a very strong disorder; the existence argument would be especially dif- 
ficult to make, since the pattern of arriving inputs to every cell would be very different. Nevertheless, in such a network, there may exist some partially coherent state in which only a fraction of cells participate at a given time (Tsodyks et al., 1993; van Vreeswijk, 1996). In vitro and in large-scale simulations (Traub, Whittington, Stanford, \& Jefferys, 1996), doublets are produced by only some interneurons in some cycles. It remains to understand how the mechanism explained in this article can work in the more disordered situation in which only a fraction of those interneurons that fire in a cycle also fire a doublet spike.

Synaptic noise in I- and E-cells could also have a drastic effect on the stability of various multispike configurations. Analysis for the two-circuit case suggests that noise in general reduces stability, although rather weakly. Thus, noise has an opposite effect from weak spatial randomness in the network, which in general makes long-range synchronization more robust.

The results of this article seem to be consistent with the recent experimental in vitro studies concerning the effect of morphine on the long-range synchrony in the hippocampal CA1 area (Whittington, Traub, Faulkner, Jefferys, \& Chettiar, 1998). These authors found that adding morphine to the system, which effectively reduces the level of inhibition in the system, causes bursting of inhibitory interneurons accompanied by lack of long-range synchrony. They suggest that this loss of synchrony may be the reason that morphine causes cognitive dysfunction clinically (Whittington et al., 1998). In our model, a decrease in $\mathrm{I} \mapsto \mathrm{I}$ inhibition corresponds to decreasing $\left|v_{0}\right|$ and/or $v_{\text {th }}$ (so less input is required to trigger firing of I-cell). This means that $a b /\left|v_{0}\right| v_{\text {th }}$ ratio would increase in equation 3.12, opening the possibility for generating higher-order multispikes in inhibitory neurons. Because such higher-order multispikes are probably unstable (or stable only in a limited parameter space), one could observe bursts without the synchrony.

Also notice that the stability regions in Figure 2 get smaller with decreasing strength of nonlocal synaptic $\mathrm{E} \mapsto \mathrm{I}$ coupling. This is in a qualitative agreement with the large-scale simulations involving multicompartmental realistic neurons of the hippocampal CA1 area (Traub, Whittington, Stanford, \& Jefferys, 1996). In those simulations it was found that reduction of $E \mapsto I$ AMPA conductance resulted in disruption of coherent global oscillations.

For large values of synaptic coupling, there is some chance that more than one multispike configuration may exist and be stable. This may be undesirable for the network if a fixed configuration is needed for downstream processing. We note that architecture-dependent synaptic depression (Thomson and Deuchars, 1994; Markram \& Tsodyks, 1996; Abbott, Sen, Varela, \& Nelson, 1997, Koch, 1997) might then help to decrease the synaptic coupling to values at which only the doublets are stable. We also note that since the period of the network oscillation depends on the extra inhibition produced by the doublet spike, modulation of the percentage of interneurons firing doublets may provide a mechanism for modulation of the period. 


\section{Appendix A}

In this appendix we show how to derive equation 3.3.

The local $l$ th I-cell receives a spike train of nonlocal excitatory cells at times $t_{l(1)}, \ldots, t_{l\left(k_{l}\right)}, \ldots, t_{l\left(n_{l}\right)}$, after which it fires, where $l(k) \Longrightarrow+k$. Solving equations 2.1 and 2.2 with an initial condition $v\left(t_{l}^{E}\right) \Longrightarrow v_{0}$, we obtain recursive formulas for the values of the potential $v(t)$

$$
\begin{array}{r}
v\left(t_{l(1)}\right) \Longrightarrow v_{0} e^{-\left(t_{l(1)}-t_{l}^{E}\right) / \tau_{m}^{i}}+a, \\
v\left(t_{l(2)}\right) \Longrightarrow\left(t_{l(1)}\right) e^{-\left(t_{l(2)}-t_{l(1)}\right) / \tau_{m}^{i}}+a, \\
\ldots \ldots \\
v\left(t_{l\left(k_{l}\right)}\right) \Longrightarrow\left(t_{l\left(k_{l}-1\right)}\right) e^{-\left(t_{l\left(k_{l}\right)}-t_{l\left(k_{l}-1\right)}\right) / \tau_{m}^{i}}+a,
\end{array}
$$

up to the time $\delta t_{l} \equiv t_{l}\left(k_{l}\right)$ when $v\left(\delta t_{l}\right)=0$. This time is given by

$$
\delta t_{l}=\tau_{m}^{i} \ln \left[\frac{\left|v_{0}\right|}{a}-\sum_{i=1}^{k_{l}-1} e^{\left(t_{l(i)}-t_{l}^{E}\right) / \tau_{m}^{i}}\right] .
$$

At that time $v(t)$ in equation 2.1 changes its sign. This causes exponential growth described by the recursive equations

$$
\begin{aligned}
& v\left(t_{l\left(k_{l}+1\right)}\right)=b \cdot e^{\left(t_{l\left(k_{l}+1\right)}-t_{l\left(k_{l}\right)}\right) / \tau_{m}^{i}}+a, \\
& v\left(t_{l\left(k_{l}+2\right)}\right) \Longrightarrow\left(t_{l\left(k_{l}+1\right)}\right) e^{\left(t_{l\left(k_{l}+2\right)}-t_{l\left(k_{l}+1\right)}\right) / \tau_{m}^{i}}+a, \\
& v\left(t_{l\left(n_{l}\right)}\right) \Longrightarrow\left(t_{l\left(n_{l}-1\right)}\right) e^{\left(t_{l\left(n_{l}\right)}-t_{l\left(n_{l}-1\right)}\right) / \tau_{m}^{i}}+a .
\end{aligned}
$$

The I-cell fires between receiving spikes enumerated as $n_{l}$ and $n_{l}+1$. Thus the time of firing $t_{f}$ is determined from a condition

$$
v\left(t_{f}\right) \Longrightarrow\left(t_{l\left(n_{l}\right)}\right) e^{\left(t_{f}-t_{l\left(n_{l}\right)}\right) / \tau_{m}^{i}} \equiv v_{t h}
$$

This yields $t_{f}=t_{l\left(n_{l}\right)}+\tau_{m}^{i} \ln \left(v_{t h} / v\left(t_{l\left(n_{l}\right)}\right)\right)$. Inserting values of $v\left(t_{l\left(n_{l}\right)}\right)$ and $t_{l\left(k_{l}\right)}$, and using the fact that $W_{l}^{I}=t_{f}-t_{l}^{E}$, we obtain equation 3.3.

We should mention that $v\left(t_{l\left(n_{l}\right)}\right) \approx v_{t h}$; hence the logarithmic term in the formula for $t_{f}$ is very small. Thus, an approximate formula for $W_{l}^{I}$ is $W_{l}^{I} \approx t_{l\left(n_{l}\right)}-t_{l}^{E}$. We will make use of this fact in appendix D. Nevertheless, one should be cautious about dropping the logarithmic term too quickly; this term is important in stability considerations, that is, in the calculation of the stability matrix $A$. 


\section{Appendix B}

In this appendix we show how to derive the $W_{l}^{E}$ function in equation 3.4.

At time $t=t_{l}^{E}$ the $l$ th E-cell fires and its potential is reset to $v_{0}(<0)$. We solve equations 2.1 and 2.3 with two inhibitory inputs coming from the local I-cell separated by time $W_{l}^{I}$ with an initial condition $v\left(t_{l}^{E}\right) \rightleftharpoons v_{0}$. For simplicity, we assume that the time $t_{0}$ when the potential of the E-cell crosses zero is larger than $t_{l}^{E}+W_{l}^{I}$. The former is determined from the condition $v\left(t_{0}\right)=0$, which produces

$$
\begin{aligned}
0 \Longrightarrow & v_{0} e^{-\left(t_{0}-t_{l}^{E}\right) / \tau_{m}^{e}}+c\left(1-e^{-\left(t_{0}-t_{l}^{E}\right) / \tau_{m}^{e}}\right)-\frac{g \tau_{s}^{i}}{\tau_{s}^{i}-\tau_{m}^{e}} \\
& \times\left[e^{-\left(t_{0}-t_{l}^{E}\right) / \tau_{s}^{i}}-e^{-\left(t_{0}-t_{l}^{E}\right) / \tau_{m}^{e}}+e^{-\left(t_{0}-t_{l}^{E}-W_{l}^{I}\right) / \tau_{s}^{i}}-e^{-\left(t_{0}-t_{l}^{E}-W_{l}^{I}\right) / \tau_{m}^{e}}\right] .
\end{aligned}
$$

In the limit $\tau_{m}^{e} / \tau_{s}^{i} \ll 1$ this equation yields

$$
t_{0}-t_{l}^{E} \approx \tau_{s}^{i} \ln \left[\frac{g}{c}\left(1+e^{W_{l}^{I} / \tau_{s}^{i}}\right)\right]
$$

and in the limit $\tau_{m}^{e} / \tau_{s}^{i} \gg 1$ (with $c / g \ll 1$ ),

$$
t_{0}-t_{l}^{E} \approx \tau_{m}^{e} \ln \left[\frac{g \tau_{s}^{i}}{c \tau_{m}^{e}}\left(1+e^{W_{l}^{I} / \tau_{m}^{e}}\right)\right]
$$

At $t=t_{0}, v(t)$ changes sign and begins to grow exponentially, reaching the threshold $v_{t h}$. Solving equations 2.1 and 2.3 as before with an initial condition $v\left(t_{0}\right)=0$, we obtain in the limit $\tau_{m}^{e} / \tau_{s}^{i} \ll 1$, the time $t_{\text {th }}$ at which $v\left(t_{t h}\right) \Longrightarrow t_{t h}$ :

$$
t_{t h}-t_{0} \approx \tau_{m}^{e} \ln \left(+\frac{v_{t h} \tau_{s}^{i}}{c \tau_{m}^{e}}\right)
$$

In the limit $\tau_{m}^{e} / \tau_{s}^{i} \gg 1$, the time $t_{t h}$ is given by

$$
t_{t h}-t_{0} \approx \tau_{m}^{e} \ln \left(1+\frac{v_{t h}}{c}\right)
$$

Since $W_{l}^{E}=t_{t h}-t_{l}^{E}-W_{l}^{I}$, we can neglect $t_{t h}-t_{0}$ part in the limit $\tau_{m}^{e} / \tau_{s}^{i} \ll 1$, which then yields

$$
W_{l}^{E} \approx t_{0}-t_{l}^{E}-W_{l}^{I}=\tau_{s}^{i} \ln \left[\frac{g}{c}\left(1+e^{-W_{l}^{I} / \tau_{s}^{i}}\right)\right]
$$

We cannot neglect $t_{t h}-t_{0}$ part in the limit $\tau_{m}^{e} / \tau_{s}^{i} \gg 1$, because both parts are 
of the same order. As a result, in this limit, we obtain

$$
W_{l}^{E} \approx \tau_{m}^{e} \ln \left[\frac{g \tau_{s}^{i}}{c \tau_{m}^{e}}\left(1+\frac{v_{t h}}{c}\right)\left[1+e^{-W_{l}^{I} / \tau_{m}^{e}}\right]\right] .
$$

Formulas B.6 and B.7 differ mainly by a substitution $\tau_{s}^{i} \leftrightarrow \tau_{m}^{e}$; this is the reason that stability diagrams in both regimes look the same. Both formulas are similar in form to that adopted by Ermentrout and Kopell (1998).

\section{Appendix C}

In this appendix we demonstrate that the probability distribution $P_{1}(x)$ with delta functions (see equation 3.6) and gaussian distribution $P_{2}=\exp [-(x-$ $\left.x_{0}\right)^{2} / 2 \zeta^{2}$ ] are equivalent in the limit $\zeta / x_{0} \ll 1$, which is the limit of weak disorder.

To prove the above statement, it is sufficient to show that $n$-moment of $x$ computed using both distributions is the same in the above limit. For the gaussian distribution we have

$$
\left\langle x^{n}\right\rangle_{P_{2}}=Z^{-1} \int_{-\infty}^{\infty} d x P_{2}(x) \cdot x^{n}=Z^{-1} \int_{-\infty}^{\infty} d x \cdot x^{n} e^{-\left(x-x_{0}\right)^{2} / 2 \zeta^{2}},
$$

where $\mathrm{Z}$ is the normalization factor. This integral can be computed using

$$
x^{n} e^{-\left(x-x_{0}\right)^{2} / 2 \zeta^{2}} \equiv e^{-x_{0}^{2} / 2 \zeta^{2}}\left(\frac{\partial}{\partial x_{0}}\right)^{x_{0}^{2} / 2 \zeta^{2}} e^{-\left(x-x_{0}\right)^{2} / 2 \zeta^{2}} .
$$

The result is

$$
\left\langle x^{n}\right\rangle_{P_{2}}=\zeta^{2 n} e^{-x_{0}^{2} / 2 \zeta^{2}}\left(\frac{\gamma}{x_{0}}\right)^{x_{0}^{2} / 2 \zeta^{2}}
$$

which yields $\langle x\rangle_{P_{2}}=x_{0},\left\langle x^{2}\right\rangle_{P_{2}}=x_{0}^{2}\left(1+\zeta^{2} / x_{0}^{2}\right),\left\langle x^{3}\right\rangle_{P_{2}}=x_{0}^{3}\left(1+3 \zeta^{2} / x_{0}^{2}\right)$, and so forth.

On the other hand, for $P_{1}$ distribution we have

$$
\begin{aligned}
\left\langle x^{n}\right\rangle_{P_{1}} & =\frac{1}{2} \int_{-\infty}^{\infty} d x \cdot x^{n}\left[\delta\left(x-x_{0}+\zeta\right)+\delta\left(x-x_{0}-\zeta\right)\right] \\
& =x_{0}^{n}\left[1+\frac{n(n-1)}{2} \frac{\zeta^{2}}{x_{0}^{2}}\right]+O\left(\zeta^{4} / x_{0}^{4}\right) .
\end{aligned}
$$

Thus, both probability distributions give the same results in the limit $\zeta / x_{0} \ll$ 1 , which in our case corresponds to the limit $\zeta N / R \ll 1$. This is the reason 
that we have used simple $P_{1}$ distribution with delta functions instead of more complicated gaussian distribution. In that way we avoided complex multidimensional integrals in equation 3.7.

Notice that our proof is valid for a random variable $x$, assuming both positive and negative values. One can also show that the above conclusion is valid for a random variable defined only on a positive interval $0<x<\infty$, although calculations in this case are more tedious.

\section{Appendix D}

In this appendix we show how to derive the average value of any function when the probability distribution is given by equations 3.5 and 3.6. We also show how to obtain the average number of inputs $k_{l}$ and $n_{l}$ needed for crossing the zero potential value and threshold, respectively.

For an arbitrary function $H\left(L_{l, l}(1), \ldots, L_{l, l(k)} ;\left\{\Delta_{l}\right\}\right)$ we want to calculate its average value $\langle H\rangle$, that is, carry out the multidimensional integrals in equation 3.7. We obtain

$$
\begin{aligned}
& \langle H\rangle=\frac{1}{2^{k} \cosh ^{k}(\zeta / \sigma)} \sum_{\alpha_{1}, \ldots, \alpha_{k}=1}^{2} H\left(\mathbf{L}^{+}+(-1)^{\alpha_{1}} \zeta, \ldots, \frac{k R}{N}+(-1)^{\alpha_{k}} \zeta ;\left\{\Delta_{l}\right\}\right) \\
& \quad \times \exp \left(-\sum_{j=1}^{k}(-1)^{\alpha_{j} \zeta / \sigma}\right),
\end{aligned}
$$

where the prefactor term comes from the normalization of the probability distribution $\rho(\{L\})$. Using this formula and the facts that $t_{l(k)}=l_{l(k)}^{E}+L_{l, l(k)}$ and $t_{k}^{E}-t_{1}^{E} \equiv \Delta_{k-1}$, we can easily write formulas for $\left\langle W^{I}\right\rangle$ and $\left\langle W^{E}\right\rangle$ based on equations 3.3 and 3.4. Their forms are as follows

$$
\begin{aligned}
\left\langle W_{l}^{I}\right\rangle= & \left.t_{l\left(n_{l}\right)}^{E}\right\rangle-\left\langle t_{l}^{E}\right\rangle+\frac{1}{2 \cosh (\zeta / \sigma)} \sum_{\alpha=1}^{2}\left[\frac{\left\langle n_{l}\right\rangle R}{N}+(-1)^{\alpha_{\zeta}}\right] \\
& \times e^{-(-1)^{\alpha} \zeta / \sigma}-\frac{\tau_{m}^{i}}{2^{n_{l}} \cosh ^{n_{l}}(\zeta / \sigma)} \times \sum_{\alpha_{1}, \ldots, \alpha_{n_{l}}=}^{2} \exp \left(-\sum_{j=1}^{n_{l}}(-1)^{\alpha_{j} \zeta / \sigma}\right) \\
& \times \ln \left(\frac{\exp \left[\left(n_{l} R / N+(-1)^{\alpha_{n_{l}}} \zeta-\delta t_{l}+\Delta_{l+n_{l}-1}-\Delta_{l-1}\right) / \tau_{m}^{i}\right]}{}\right. \\
& +\frac{a}{v_{t h}} \sum_{i=k_{l}}^{n_{l}} \exp \left[\left(\left(n_{l}-i\right) R / N+\left[(-1)^{\alpha_{n_{l}}}-(-1)^{\alpha_{i}}\right] \zeta\right.\right. \\
& \left.\left.\left.+\Delta_{l+n_{l}-1}-\Delta_{l+i-1}\right) / \tau_{m}^{i}\right]\right)
\end{aligned}
$$


and

$$
\begin{aligned}
& \left\langle W_{l}^{E}\right\rangle=\frac{\tau_{s}^{i}}{2^{n_{l}} \cosh ^{n_{l}}(\zeta / \sigma)} \sum_{\alpha_{1}, \ldots, \alpha_{n_{l}}=1}^{2} \exp \left(-\sum_{j=1}^{n_{l}}(-1)^{\alpha_{j}} \zeta / \sigma\right) \\
& \times \ln \left[\frac { g } { c } \left(+\exp \left[-W_{l}^{I} \frac{\not r}{\mathbf{L}}+(-1)^{\alpha_{1}} \zeta, \ldots, \frac{n_{l} R}{N}\right.\right.\right.
\end{aligned}
$$

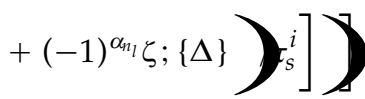

where $\delta t_{l}$ is given by equation A.2.

We can also write the sum $\left.\left\langle W^{I}\right\rangle+\left\langle W^{E}\right\rangle=\tau_{s}^{i} \ln \left[\frac{g}{c}\left(1+\exp \left(W_{l}^{I} / \tau_{s}^{i}\right)\right)\right]\right\rangle$, which is equation 3.11 in the text. Note that $\left\langle W^{E}\right\rangle$ and $\left\langle W^{I}\right\rangle+\left\langle W^{E}\right\rangle$ differ only by the sign of $W^{I}$ present in the logarithmic term; compare equations D.3 and 3.11 .

We can also derive the average value of $k_{l}$ and $n_{l}$, defined below, in the synchronous state using equation D.1. From equation A.1, we obtain

$$
\left\langle v\left(t_{l\left(k_{l}\right)}\right)\right\rangle=v_{0}\left\langle e^{\left.-L_{l, l\left(k_{l}\right)}\right) \tau_{m}^{i}}\right\rangle+a \sum_{i=1}^{k_{l}}\left\langle e^{-\left(L_{l, l\left(k_{l}\right)}-L_{l, l(i)}\right) / \tau_{m}^{i}}\right\rangle
$$

for the synchronous state with $\Delta_{1}=\cdots=\Delta_{N-1}=0$. In deriving equation D.4, we again have used the relation $t_{l(k)} t_{l(k)}^{E}+L_{l, l(k)}$. Invoking equation D.1 yields

$$
\left\langle e^{-L_{l, l\left(k_{l}\right) / \tau_{m}^{i}}}\right\rangle=\frac{\cosh \left(\frac{\zeta}{\sigma}+\frac{\zeta}{\tau_{m}^{i}}\right)}{\cosh \frac{\zeta}{\sigma}}\left\langle e^{-k_{l} R / N \tau_{m}^{i}}\right\rangle
$$

and

$$
\left\langle e^{-\left(L_{l, l\left(k_{l}\right)}-L_{l, l(i)}\right) / \tau_{m}^{i}}\right\rangle=\frac{\cosh \left(\frac{\zeta}{\sigma}+\frac{\zeta}{\tau_{m}^{i}}\right) \cosh \left(\frac{\zeta}{\sigma}-\frac{\zeta}{\tau_{m}^{i}}\right)}{\cosh ^{2} \frac{\zeta}{\sigma}}\left\langle e^{-\left(k_{l}-i\right) R / N \tau_{m}^{i}}\right\rangle .
$$

Condition $\left\langle v\left(t_{l\left(k_{l}\right)}\right)\right\rangle=0$ determines the average value of $k_{l}$ denoted by $k$, which we define as $k=\left(N \tau_{m}^{i} / R\right) \ln \left[\left\langle\exp \left(k_{l} R / N \tau_{m}^{i}\right)\right\rangle\right]$. The formula for $k$ takes the form

$$
k=\frac{N \tau_{m}^{i}}{R} \ln \left(\begin{array}{c}
{\left[\left|v_{0}\right|\left(1-e^{-R / N \tau_{m}^{i}}\right) \cosh \frac{\zeta}{\sigma}\right.} \\
\left.+a \cosh \left(\frac{\zeta}{\sigma}-\frac{\zeta}{\tau_{m}^{i}}\right)\right] \cosh \left(\frac{\zeta}{\sigma}+\frac{\zeta}{\tau_{m}^{i}}\right) \\
a\left[\cosh ^{2} \frac{\zeta}{\sigma}+e^{-R / N \tau_{m}^{i}} \sinh ^{2} \frac{\zeta}{\tau_{m}^{i}}\right]
\end{array}\right) .
$$


In a similar manner we obtain the average value of $n_{l}$, denoted by $n$, from the condition $\left\langle v\left(t_{l\left(n_{l}\right)}\right)\right\rangle \Longrightarrow v_{t h}$. Our $n$, defined as $n=\left(N \tau_{m}^{i} / R\right) \ln \left[\left\langle\exp \left(n_{l} R / N \tau_{m}^{i}\right)\right\rangle\right]$, takes the form

$$
\begin{aligned}
n=\frac{N \tau_{m}^{i}}{R} \ln \left(\begin{array}{l}
{\left[\left(v_{t h}-a\right)\left(1-e^{-R / N \tau_{m}^{i}}\right) \cosh ^{2} \frac{\zeta}{\sigma}\right.} \\
\left.+a \cosh \left(\frac{\zeta}{\sigma}+\frac{\zeta}{\tau_{m}^{i}}\right) \cosh \left(\frac{\zeta}{\sigma}-\frac{\zeta}{\tau_{m}^{i}}\right)\right] \\
{\left[b+(a-b) e^{-R / N \tau_{m}^{i}}\right] \cosh \left(\frac{\zeta}{\sigma}-\frac{\zeta}{\tau_{m}^{l}}\right)}
\end{array}\right. \\
\left.\times \frac{\left[\left|v_{0}\right|\left(1-e^{-R / N \tau_{m}^{i}}\right) \cosh \frac{\zeta}{\sigma}+a \cosh \left(\frac{\zeta}{\sigma}-\frac{\zeta}{\tau_{m}^{l}}\right)\right]}{a\left[\cosh ^{2} \frac{\zeta}{\sigma}+e^{-R / N \tau_{m}^{i}} \sinh ^{2} \frac{\zeta}{\tau_{m}^{l}}\right]}\right) .
\end{aligned}
$$

From the average values $k$ and $n$, we can compute $\left\langle W_{l}^{I}(\{\Delta=0\})\right\rangle$ of a synchronous state depending only on the parameters of the network architecture. This function plays an important role in the discussion about stability.

Making use of the fact that the fourth, logarithmic term in equation D.2 is small (cf. appendix A), we can neglect it. Thus, in the synchronous state, equation D.2 has the approximate form

$$
\left\langle W_{l}^{I}\right\rangle \approx \frac{\left\langle n_{l}\right\rangle R}{N}-\zeta \tanh \frac{\zeta}{\sigma} .
$$

We expect that for large $n_{l}$, its higher-order cumulants are small, so the relation $\left\langle n_{l}\right\rangle \approx n$ holds. Using this fact, we can substitute $n$ for $\left\langle n_{l}\right\rangle$ into the above equation. We obtain

$$
\begin{aligned}
& \left\langle W^{I}\right\rangle \approx \tau_{m}^{i} \ln \left(\begin{array}{c}
{\left[\left(v_{t h}-a\right)\left(1-e^{-R / N \tau_{m}^{i}}\right) \cosh ^{2} \frac{\zeta}{\sigma}\right.} \\
\left.+a \cosh \left(\frac{\zeta}{\sigma}+\frac{\zeta}{\tau_{m}^{l}}\right) \cosh \left(\frac{\zeta}{\sigma}-\frac{\zeta}{\tau_{m}^{l}}\right)\right]
\end{array}\right. \\
& \left.\times \frac{\left[\left|v_{0}\right|\left(1-e^{-R / N \tau_{m}^{i}}\right) \cosh \frac{\zeta}{\sigma}\right.}{a\left[\cosh ^{2} \frac{\zeta}{\sigma}+e^{-R / N \tau_{m}^{i}} \sinh ^{2} \frac{\zeta}{\tau_{m}^{l}}\right]}\right)-\zeta \tanh \frac{\zeta}{\sigma} .
\end{aligned}
$$

Note that $\left\langle W^{I}\right\rangle$ is of the order of the membrane time constant $\tau_{m}^{i}$ and additionally that $\left\langle W^{I}\right\rangle$ can be arbitrarily small provided the disorder parameter $\zeta$ is sufficiently large. 


\section{Appendix E}

In this appendix we sketch how to compute the stability diagrams depicted in Figure 2, as well as how to derive equation 3.13. Formulas below are provided only in the regime $\tau_{m}^{e} / \tau_{s}^{i} \ll 1$. In the opposite regime, $\tau_{m}^{e} / \tau_{s}^{i} \gg 1$, formulas are the same with a substitution $\tau_{s}^{i} \mapsto \tau_{m}^{e}$.

According to the Gershgorin theorem (Horn \& Johnson, 1985), the lth eigenvalue $\lambda_{l}$ of the stability matrix $A$ lies in a disk such that

$$
\left|\lambda_{l}-A_{l, l}\right| \leq \sum_{m \neq 1}\left|A_{l, m}\right|
$$

where $A_{l, m} \equiv \partial \bar{\Delta}_{l} /\left.\partial \Delta_{m}\right|_{\Delta=\theta}$. Using the inequality $\left|\lambda_{l}-A_{l, l}\right| \geq\left|\lambda_{l}\right|-\left|A_{l, l}\right|$, we obtain $\left|\lambda_{l}\right| \leq \sum_{m=1}^{N-1}\left|A_{l, m}\right|$. The system is stable when $\left|\lambda_{l}\right|<1$ for every $l$. Thus, a sufficient condition for stability is

$$
\sum_{m=1}^{N-1}\left|A_{l, m}\right|<1
$$

for every $l=1, \ldots, N-1$. After some algebra the sum in equation E.2 can be found as

$$
\begin{aligned}
\sum_{m=1}^{N-1}\left|A_{l, m}\right|=\frac{2}{1+e^{-n R / N \tau_{s}^{i}}} & \left(+\frac{b\left|v_{0}\right|}{a v_{t h}}\left\langle e^{\left[L_{l, l\left(n_{l}\right)}-2 L_{\left.l, l\left(k_{l}\right)\right] / \tau_{m}^{i}}^{i}\right\rangle-\frac{b}{v_{t h}}\left\langle e^{\left[L_{l, l\left(n_{l}\right)}-L_{l, l\left(k_{l}\right)}\right] / \tau_{m}^{i}}\right\rangle}\right.\right. \\
& \left.+\frac{1}{2} \frac{b}{v_{t h}}\left\langle e^{\left[L_{l, l\left(n_{l}\right)}-L_{l, l\left(k_{l}\right)}\right] / \tau_{m}^{i}}\right\rangle-e^{-n R / N \tau_{s}^{i}}\right)
\end{aligned}
$$

Next, invoking equations D.1, D.5, and D.6, we can find the averages in the above equation. Combining all this, equation E. 2 decomposes into two complementary cases, each described by a pair of inequalities, due to the modulus present in equation E.3. We discuss the first case in detail. Later we comment on the complementary second case. The inequalities for the first case are given by

$$
e^{(n-k) R / N \tau_{m}^{i}}>\frac{v_{t h}}{b} \frac{\cosh ^{2} \frac{\zeta}{\sigma}}{\cosh \left(\frac{\zeta}{\sigma}+\frac{\zeta}{\tau_{m}^{i}}\right) \cosh \left(\frac{\zeta}{\sigma}-\frac{\zeta}{\tau_{m}^{i}}\right)} e^{-n R / N \tau_{s}^{i}}
$$

and

$$
\begin{aligned}
e^{n R / N \tau_{s}^{i}}[ & +2 \frac{b\left|v_{0}\right|}{a v_{t h}} \frac{\cosh \left(\frac{\zeta}{\sigma}+2 \frac{\zeta}{\tau_{m}^{i}}\right) \cosh \left(\frac{\zeta}{\sigma}-\frac{\zeta}{\tau_{m}^{l}}\right)}{\cosh ^{2} \frac{\zeta}{\sigma}} e^{(n-2 k) R / N \tau_{m}^{i}} \\
& \left.-\frac{b}{v_{t h}} \frac{\cosh \left(\frac{\zeta}{\sigma}+\frac{\zeta}{\tau_{m}^{i}}\right) \cosh \left(\frac{\zeta}{\sigma}-\frac{\zeta}{\tau_{m}^{i}}\right)}{\cosh ^{2} \frac{\zeta}{\sigma}} e^{(n-k) R / N \tau_{m}^{i}}\right]<2,
\end{aligned}
$$


where $n$ and $k$ are given by equations D.7 and D.8. Inequalities E.4 and E.5 must be satisfied simultaneously.

Notice that after insertion of equations for $k$ and $n, \exp \left(n R / N \tau_{s}^{i}\right)$ becomes a function of the ratios $\tau_{m}^{i} / \tau_{s}^{i}$ and $R / N \tau_{m}^{i}$, and $\exp \left(n R / N \tau_{m}^{i}\right)$ becomes a function of $R / N \tau_{m}^{i}$ ratio for given other parameters. This says that we can compute a sufficient condition for the stability in terms of $\tau_{m}^{i} / \tau_{s}^{i}$ and $R / N \tau_{m}^{i}$.

In the ordered network case for $\zeta \Longrightarrow$, equations E.4 and E.5 reduce to

$$
\tau_{m}^{i} / \tau_{s}^{i}>\frac{1}{K} \ln \left(\frac{v_{t h}\left[b+(a-b) e^{-R / N \tau_{m}^{i}}\right]}{b\left[\left(v_{t h}-a\right)\left(1-e^{-R / N \tau_{m}^{i}}\right)+a\right]}\right)
$$

and

$$
\begin{aligned}
\tau_{m}^{i} / \tau_{s}^{i}<-\frac{1}{K} \ln \left(\frac{1}{2}[1\right. & +\frac{b\left[\left(v_{t h}-a\right)\left(1-e^{-R / N \tau_{m}^{i}}\right)+a\right]}{v_{t h}\left[b+(a-b) e^{-R / N} \tau_{m}^{i}\right]} \\
& \left.\left.\times \frac{\left[\left|v_{0}\right|\left(1+e^{-R / N \tau_{m}^{i}}\right)-a\right]}{\left[\left|v_{0}\right|\left(1-e^{-R / N \tau_{m}^{i}}\right)+a\right]}\right]\right),
\end{aligned}
$$

with

$$
K=\ln \left(\frac{\left[\left(v_{t h}-a\right)\left(1-e^{-R / N \tau_{m}^{i}}\right)+a\right]\left[\left|v_{0}\right|\left(1-e^{-R / N \tau_{m}^{i}}\right)+a\right]}{a\left[b+(a-b) e^{-R / N \tau_{m}^{i}}\right]}\right) .
$$

The two curves on the right-hand side of equations E.6 and E.7 cross in the point with coordinates

$$
R / N \tau_{m}^{i} \approx \ln \left[(a+2 b)\left|v_{0}\right| / 2 a b\right]
$$

and

$$
\tau_{m}^{i} / \tau_{s}^{i} \approx-\ln \left[1-2 a^{2} /(a+2 b)\left|v_{0}\right|\right] / \ln \left(v_{t h}\left|v_{0}\right| / a b\right),
$$

denoted as $x_{0}$ and $y_{0}$ in Figure 2, respectively. Above this value of $R / N \tau_{m}^{i}$ the set of inequalities E.6 and E.7 is satisfied.

The complementary case differs from the first by inverting the inequality sign in equation E.4 and multiplying the third term in the brackets in equation E.5 by 3 and setting the right side of this inequality equal to zero. In this case we also obtain the stability region for $R / N \tau_{m}^{i}$ greater than the value given by equation E.9. Combining these two cases, we find the stability region as that shadowed in Figure $2 \mathrm{~A}$. Notice that the ratio $\tau_{m}^{i} / \tau_{s}^{i}$ must be small in order to preserve stability. 
The disordered network case with $\zeta>0$ is more complicated algebraically to analyze. In this case, there also exists some minimum value of $R / N \tau_{m}^{i}$ of the order of that in equation E.9 for weak disorder, below which the doublet configuration is unstable.

The disordered case differs from the ordered case by the appearance of a vertical asymptote in Figure $2 \mathrm{~B}$, which crosses the horizontal axis at $\left(R / N \tau_{m}^{i}\right)_{c r}$ given by equation 3.13. The reason for the appearance of the asymptote is the fact that the term in the bracket in equation E.5 can now change sign. For small $R / N \tau_{m}^{i}$, the bracket term is positive, whereas for $R / N \tau_{m}^{i} \mapsto \infty$, it is negative. This does not happen for the ordered network; in this case, the bracket term is always positive. When the bracket term is negative, inequality E.5 is satisfied for every $\tau_{m}^{i} / \tau_{s}^{i}$ (recall that this ratio comes from $\exp \left(n R / N \tau_{s}^{i}\right)$ term).

Putting the bracket term equal to zero and substituting for $n$ and $k$ values represented by equations D.7 and D.8 determines the vertical asymptote in Figure 2B. In the limit $R / N \tau_{m}^{i} \gg \zeta / \tau_{m}^{i} \gg 1$ we obtain $\left(R / N \tau_{m}^{i}\right)_{c r}$ given by equation 3.13. Notice that for the ordered network with $\zeta \Longrightarrow$, our asymptote shifts to infinity (that is, it disappears), which corresponds to decreasing the stability region.

For the triplet configuration, analysis shows that in the ordered network case, the condition E. 2 cannot be satisfied for any $\tau_{m}^{i} / \tau_{s}^{i}$ and $R / N \tau_{m}^{i}$. In the disordered network case, we can show that there is some stability region for $R / N \tau_{m}^{i} \gg 1$.

\section{Acknowledgments}

We thank B. Ermentrout, C. Chow, and particularly R. Traub for discussions. The work was supported by NSF DMS 9706694 and NIMH R01 MH47150. J. K. thanks the Fulbright Foundation for partial support.

\section{References}

Abbott, L. F., Sen, K., Varela, J. A., \& Nelson, S. B. (1997). Synaptic depression and cortical gain control. Science, 275, 220-224.

Abbott, L. F., \& van Vreeswijk, C. (1993). Asynchronous states in network of pulse-coupled oscillators. Physical Review E, 48, 1483-1490.

Ahissar, E., Vaadia, E., Ahissar, M., Bergman, H., Arieli, A., \& Abeles, M. (1992). Dependence of cortical plasticity on correlated activity of single neurons and on behavioral context. Science, 257, 1412-1415.

Amit, D. J., Gutfreund, H., \& Sompolinsky, H. (1985). Spin-glass models of neural networks. Physical Review A, 32, 1007-1018.

Andersen, P., Silfvenius, H., Sundberg, S., Sveen, O., \& Wigstrom, H. (1978). Functional characteristics of unmyelinated fibres in the hippocampal cortex. Brain Res., 144, 11-18. 
Bliss, T. V. P., \& Collingride, G. L. (1993). A synaptic model of memory: Longterm potentiation in the hippocampus. Nature, 361, 31-34.

Bragin, A., Jando, G., Nadasdy, Z., Hetke, J., Wise, K., \& Buzsaki, G. (1995). Gamma $(40-100 \mathrm{~Hz})$ oscillations in the hippocampus of the behaving rat. J. Neurosci., 15(1), 47-60.

Bressler, S. L., Coppola, R., \& Nakamura, R. (1993). Episodic multiregional cortical coherence at multiple frequencies during visual task performance. Nature, 366, 153-156.

Buhl, E. H., Halasy, K., \& Somogi, P. (1994). Diverse sources of hippocampal unitary inhibitory postsynaptic potentials and the number of synaptic release sites. Nature, 368, 823-828.

Bush, P., \& Sejnowski, T. (1996). Inhibition synchronizes sparsely connected cortical neurons within and between columns of realistic network models. $J$. Comp. Neurosci., 3, 91-110.

Campbell, S. R., \& Wang, D. (1998). Relaxation oscillators with time delay coupling. Preprint.

Chow, C. C., White, J. A., Ritt, J., \& Kopell, N. (1998). Frequency control in synchronized networks of inhibitory neurons. J. Comp. Neurosci., 5, 407-420.

Crook, S. M., Ermentrout, G. B., Vanier, M. C., \& Bower, J. M. (1997). The role of axonal delay in the synchronization of networks of coupled cortical oscillators. J. Comp. Neurosci., 4, 161-172.

Engel, A. K., Konig, P., \& Singer, W. (1991). Direct physiological evidence for scene segmentation by temporal coding. Proc. Natl. Acad. Sci. USA, 88, 91369140.

Engel, A. K., Kreiter, A. K., Konig, P., \& Singer, W. (1991). Synchronization of oscillatory neuronal responses between striate and extrastriate visual cortical areas of the cat. Proc. Natl. Acad. Sci. USA, 88, 6048-6052.

Ermentrout, G. B. (1996). Type I membranes, phase resetting curves and synchrony. Neural Comp., 8, 979-1002.

Ermentrout, G. B., \& Kopell, N. (1998). Fine structure of neural spiking and synchronization in the presence of conduction delays. Proc. Natl. Acad. Sci. USA, 95, 1259-1264.

Ernst, U., Pawelzik, K., \& Geisel, T. (1995). Synchronization induced by temporal delays in pulse-coupled oscillators. Physical Review Lett., 74, 15701573.

Freund, T. F., \& Buzsaki, G. (1996). Interneurons of the hippocampus. Hippocampus, 6, 347-470.

Fries, P., Roelfsema, P. R., Engel, A. K., Konig, P., \& Singer, W. (1997). Synchronization of oscillatory responses in visual cortex correlates with perception in interocular rivalry. Proc. Natl. Acad. Sci. USA, 94, 12699 12704.

Friesen, W. (1994). Reciprocal inhibition, a mechanism underlying oscillatory animal movements. Neurosci. Behavior, 18, 547-553.

Gerstner, W. (1995). Time structure of the activity in neural networks models. Physical Review E, 51, 738-758.

Gerstner, W., van Hemmen, J. L., \& Cowan, J. D. (1996). What matters in neuronal locking? Neural Comp., 8, 1653-1676. 
Gray, C. M., Konig, P., Engel, A. K., \& Singer, W. (1989). Oscillatory responses in cat visual cortex exhibit inter-columnar synchronization which reflects global stimulus properties. Nature, 338, 334-337.

Hebb, D. O. (1949). The organization of behavior. New York: Wiley.

Hertz, J., Krogh, A., \& Palmer, R. G. (1991). Introduction to the theory of neural computations. Redwood City, CA: Addison-Wesley.

Hopfield, J. J. (1984). Neurons with graded response have collective computational properties like those of two-state neurons. Proc. Natl. Acad. Sci. USA, 81, 3088-3092.

Horn, R. A., \& Johnson, C. A. (1985). Matrix analysis. Cambridge: Cambridge University Press.

Jefferys, J. G. R., Traub, R. D., \& Whittington, M. A. (1996). Neuronal networks for induced " $40 \mathrm{~Hz}$ " rhythms. Trends Neurosci., 19, 202-208.

Joliot, M., Ribary, U., \& Llinas, R. (1994). Human oscillatory brain activity near $40 \mathrm{~Hz}$ coexists with cognitive temporal binding. Proc. Natl. Acad. Sci. USA, 91, 11748-11751.

Knight, B. W. (1972). Dynamics of encoding in a population of neurons. J. Gen. Physiol., 59, 734-766.

Knowles, W., \& Schwartzkroin, P. (1981). Local circuit synaptic interactions in hippocampal brain slices. J. Neurosci., 1, 318-322.

Koch, C. (1997). Computation and the single neuron. Nature, 385, 207-210.

Konig, P., Engel, A. K., \& Singer, W. (1995). Relation between oscillatory activity and long-range synchronization in cat visual cortex. Proc. Natl. Acad. Sci. USA, 92, 290-294.

Konig, P., \& Schillen, T. B. (1991). Stimulus-dependent assembly formation of oscillatory responses: I. Synchronization. Neural Comp., 3, 155-166.

Llinas, R., \& Ribary, U. (1993). Coherent 40-Hz oscillation characterizes dream state in humans. Proc. Natl. Acad. Sci. USA, 90, 2078-2081.

Lytton, W., \& Sejnowski, T. (1991). Simulations of cortical pyramidal neurons synchronized by inhibitory neurons. J. Neurophysiol., 66, 1059-1097.

Magee, J. C., \& Johnston, D. (1997). A synaptically controlled, associative signal for Hebbian plasticity in hippocampal neurons. Science, 275, 209213.

Markram, H., Lubke, J., Frotscher, M., \& Sakmann, B. (1997). Regulation of synaptic efficacy by coincidence of postsynaptic APs and EPSPs. Science, 275, 213-215.

Markram, H., \& Tsodyks, M. (1996). Redistribution of synaptic efficacy between neocortical pyramidal neurons. Nature, 382, 807-810.

McCullough, W. S., \& Pitts, W. (1943). A logical calculus of the ideas immanent in nervous activity. Bull. Math. Biophys., 5, 115-133.

Rieke, F., Warland, D., de Ruyter van Steveninck, R., \& Bialek, W. (1997). Spikes: Exploring the neural code. Cambridge, MA: MIT Press.

Roelfsema, P. R., Engel, A. K., Konig, P., \& Singer, W. (1997). Visuomotor integration is associated with zero time-lag synchronization among cortical areas. Nature, 385, 157-161.

Singer, W., \& Gray, C. M. (1995). Visual feature integration and the temporal correlation hypothesis. Annu. Rev. Neurosci., 18, 555-586. 
Steriade, M., Amzica, F., \& Contreras, D. (1996). Synchronization of fast (30-40 $\mathrm{Hz}$ ) spontaneous cortical rhythms during brain activation. J. Neurosci., 16(1), 392-417.

Stopfer, M., Bhagavan, S., Smith, B. H., \& Laurent, G. (1997). Impaired odour discrimination on desynchronization of odour-encoding neural assemblies. Nature, 390, 70-74.

Tamamaki, N., \& Nojyo, T. (1990). Disposition of the slab-like modules formed by axon branches originating from single CA1 pyramidal neurons in the rat hippocampus. J. Comp. Neurol., 291, 509-519.

Terman, D., Kopell, N., \& Bose, A. (1998). Dynamics of two mutually coupled slow inhibitory neurons. Physica D, 117, 241-275.

Terman, D., \& Rubin, J. (1998). Geometric analysis of population rhythms in synaptically coupled neuronal networks. Neural Comput. (in press).

Thomson, A. M., \& Deuchars, J. (1994). Temporal and spatial properties of local circuits in neocortex. Trends Neurosci., 17, 119-126.

Traub, R. D., Jefferys, J. G. R., \& Whittington, M. A. (1999). Fast oscillations in cortical circuits. Cambridge, MA: MIT Press.

Traub, R. D., \& Miles, R. (1991). Neuronal networks of the hippocampus. Cambridge: Cambridge University Press.

Traub, R. D., \& Miles, R. (1995). Pyramidal cell-to-inhibitory cell spike transduction explicable by active dendritic conductances in inhibitory cell. J. Comp. Neurosci., 2, 291-298.

Traub, R. D., Whittington, M. A., Buhl, E. H., Jefferys, J. G. R, \& Faulkner, H. J. (1999). On the mechanism of the gamma-beta frequency shift in neuronal oscillations induced in rat hippocampal slices by tetanic stimulation. J. Neurosci., 19, 1088-1105.

Traub, R. D., Whittington, M. A., Colling, S., Buzsaki, G., \& Jefferys, J. (1996). Analysis of gamma rhythms in the rat hippocampus in vitro and in vivo. $J$. Physiol., 493, 471-484.

Traub, R. D., Whittington, M. A., Stanford, M., \& Jefferys, J. G. R. (1996). A mechanism for generation of long-range synchronous fast oscillations in the cortex. Nature, 383, 621-624.

Tsodyks, M., Mitkov, I., \& Sompolinsky, H. (1993). Pattern of synchrony in inhomogeneous networks of oscillators with pulse interactions. Physical Review Lett., 71, 1280-1283.

Usher, M., Stemmler, M., Koch, C., \& Olami, Z. (1994). Network amplification of local fluctuations causes high spike rate variability, fractal firing patterns and oscillatory local field potentials. Neural Comp., 6, 795-836.

Vaadia, E., Haalman, I., Abeles, M., Bergman, H., Prut, Y., Slovin, H., \& Aertsen, A. (1995). Dynamics of neuronal interactions in monkey cortex in relation to behavioural events. Nature, 373, 515-518.

van Vreeswijk, C. (1996). Partial synchrony in populations of pulse-coupled oscillators. Physical Review E, 54, 5522-5537.

van Vreeswijk, C., Abbott, L. F., \& Ermentrout, G.B. (1994). When inhibition not excitation synchronizes neuronal firing. J. Comp. Neurosci., 1, 313-321.

Wang, X. J., \& Buzsaki, G. (1996). Gamma oscillations by synaptic inhibition in an interneuronal network. J. Neurosci., 16, 6402-6413. 
Wang, X. J., \& Rinzel, J. (1992). Alternating and synchronous rhythms in reciprocally inhibitory model neurons. Neural Comp., 4, 84-97.

Wang, X. J., \& Rinzel, J. (1993). Spindle rhythmicity in the reticularis thalami nucleus: Synchronization among mutually inhibitory neurons. Neuroscience, $53,899-904$.

White, J., Chow, C., Ritt, J., Soto, C., \& Kopell, N. (1998). Synchronous oscillation in heterogeneous, mutually inhibited neurons. J. Comp. Neurosci., 5, 5-16.

Whittington, M. A., Stanford, I. M., Colling, S. B., Jefferys, J. G. R., \& Traub, R. D. (1997). Spatiotemporal patterns of $\gamma$ frequency oscillations tetanically induced in the rat hippocampal slice. J. Physiol., 502, 591-607.

Whittington, M. A., Traub, R. D., Faulkner, H. J., Jefferys, J. G. R., \& Chettiar, K. (1998). Morphine disrupts long-range synchrony of gamma oscillations in hippocampal slices. Proc. Natl. Acad. Sci. USA, 95, 5807-5811.

Whittington, M. A., Traub, R. D., \& Jefferys, J. G. R. (1995). Synchronized oscillations in interneuron networks driven by metabotropic glutamate receptor activation. Nature, 373, 612-615.

Received January 11, 1999; accepted September 10, 1999. 